\title{
Perspective
}

PERSPECTIVE Actualité en histoire de l'art

1 | 2018

Actualité en histoire de l'art

\section{Culture visuelle du jeu sportif dans la première modernité}

Visual Culture of Sporting Game in the First Modern Era

Visuelle Kultur des Sportspiels in der frühen Neuzeit

Cultura visiva del gioco sportivo nella prima modernità

Cultura visual de los juegos deportivos en la primera modernidad

\section{Antonella Fenech Kroke}

\section{(Q) OpenEdition}

Journals

Édition électronique

URL : http://journals.openedition.org/perspective/9411

DOI : 10.4000/perspective.9411

ISSN : 2269-7721

Éditeur

Institut national d'histoire de l'art

Édition imprimée

Date de publication : 30 juin 2018

Pagination : 109-128

ISBN : 978-2-917902-46-2

ISSN : 1777-7852

\section{Référence électronique}

Antonella Fenech Kroke, "Culture visuelle du jeu sportif dans la première modernité », Perspective [En ligne], 1 | 2018, mis en ligne le 31 décembre 2018, consulté le 01 octobre 2020. URL : http:// journals.openedition.org/perspective/9411 ; DOI : https://doi.org/10.4000/perspective.9411 


\title{
Culture visuelle du jeu sportif dans la première modernité
}

\author{
Si véritablement le jeu est un ressort primordial de civilisation, \\ il ne se peut faire que ses significations secondes ne se révèlent pas instructives ${ }^{1}$.
}

Dans le monde hyper-iconique et hyper-ludique qui est le nôtre, innombrables sont les manifestations du lien que les jeux, dans une acception générale, entretiennent avec les images et qui témoignent de l'impact social, de la force politique et de l'efficacité économique d'une telle association. Si cette relation est très ancienne, elle connaît un renouvellement profond en Europe, entre le XV et la fin du XVII ${ }^{e}$ siècle. À bien des égards, les jeux sont en effet un élément constitutif de la culture de cette époque où l'on repense la valeur du temps et son usage; où l'on assiste à l'émergence de la notion de passe-temps et où l'on en formalise le rôle dans la vie sociale et individuelle ; où l'on reformule l'articulation entre individus, structures sociales et espaces (BURKE, 1995 ; ARCANGELI, 2003). Questionner la relation entre culture visuelle et jeux signifie alors réorienter les réflexions sur la centralité du phénomène ludique dans les processus culturels, en le reconsidérant au prisme de l'histoire des images et des arts. Si entre la fin du Moyen Âge et la première modernité on assiste à un moment d'effervescence ludique, cette effervescence concerne aussi la production d'images figurant les jeux.

Depuis le milieu du Xx ${ }^{e}$ siècle, la littérature scientifique s'est penchée sur le phénomène particulier qu'est le jeu, sur la classification et l'étude historique des pratiques ludiques, rendant compte de la complexité de la définition même du jeu et de l'hétérogénéité de sa culture. Deux orientations caractérisent cette littérature : la première s'est donnée pour tâche d'élaborer une définition du jeu en général ; la seconde a, en revanche, abordé la question d'un point de vue historique. Avec son Homo ludens, Johan Huizinga a proposé une analyse globale des tensions que le jeu entretient avec d'autres pratiques des sociétés et des cultures, et a donné au jeu le statut d'objet historiographique (HUIZINGA, [1938] 1988). Héritier et à la fois critique de cette conception universaliste, Roger Caillois a fondé sa relecture des phénomènes ludiques sur la classification des jeux comme des activités « délimitées » et comme des formes de comportement spécifiques (CAILLOIS, 1958 et 1967). Ces perspectives ont posé les bases d'une réflexion plus articulée qui s'est amplifiée à partir de la seconde moitié du $\mathrm{XX}^{\mathrm{e}}$ siècle. Distinctes en raison

\footnotetext{
Antonella Fenech Kroke est chargée de recherche au CNRS, Centre André Chastel (UMR 8150). Ancienne pensionnaire de I'Académie de France à Rome, ses recherches ont porté principalement sur la culture artistique et les productions visuelles (Italie, $X V l^{\mathrm{e}}$ siècle) dans leur dimension politique et culturelle. Actuellement elle s'intéresse aux pratiques ludiques dans les images et dans les sociétés de la première modernité ainsi qu'aux images de l'inversion et du renversement corporels (Corps à contresens, en préparation).
} 
de leurs perspectives disciplinaires, de nombreuses contributions ${ }^{2}$ ont révélé la nécessité de considérer la pluralité des acceptions du jeu et de ne pas parler du jeu comme d'une catégorie univoque. Plus récemment, les historiens ont aussi investi ce champ d'étude : parallèlement aux travaux de Gherardo Ortalli abordant la question à la lumière de l'histoire culturelle, sociale et économique ${ }^{3}$, des perspectives analogues ont été adoptées par des spécialistes de la modernité. Or, ces travaux ${ }^{4}$ ont souvent proposé des synthèses englobant des pratiques ludiques dont la nature est profondément différente : les jeux d'enfants, les jeux de hasard et de société, ou encore les jeux d'exercice, c'est-à-dire des activités ludiques caractérisées par la performance physique, la maîtrise de techniques corporelles et, très souvent, la compétition. En somme, ce que l'on appelle aujourd'hui les sports.

C'est précisément aux images des pratiques sportives prémodernes que sont consacrées ces pages. D'entrée de jeu, il convient toutefois de rappeler qu'une controverse anime la littérature (MCCLELLAND, 2009) : il serait en effet problématique d'employer le terme "sport " pour désigner les jeux physiques d'avant 1750 . Si un certain nombre d'historiens admettent à la fois cette terminologie et la continuité entre sports actuels et jeux d'exercice anciens ${ }^{5}$, de nombreux autres pensent que ces derniers sont, certes, des « activités physiques autotéliques compétitives " (GUTTMANN, 1986, p. 4) mais que leur nature diffèrerait des sports - comme pratiques organisées, séculaires, spécialisées et normalisées visant à la quantification de performances et de records - dont l'essor n'aurait lieu qu'à partir de la fin du XVIII siècle ${ }^{6}$. Or, la présente étude envisage de repenser, par les images, les pratiques d'exercice prémodernes précisément comme des sports dont les enjeux, les fonctions et les normes les distinguent d'autres formes ludiques (jeux de hasard, de société...). Parce que ces activités (individuelles et collectives) ont été le sujet de très nombreuses images, il importe de saisir leur portée dans l'analyse historique du phénomène sportif7.

À une époque où la recherche tire parti de la porosité des disciplines, l'étude de ces images est l'occasion d'interroger les conditions et les effets de la rencontre entre culture visuelle et histoire de l'art, histoire, histoire sociale et anthropologie culturelle. Plusieurs questions traversent l'étude de tels objets. Que donnent-ils à voir de la culture ludique et de la culture visuelle prémodernes, tant à l'historien de l'art et des images qu'à l'historien ? Comment articulent-ils pratiques et histoire du corps ? La répartition sociale que les images de ces activités sous-entendent concorde-elle avec les autres formes de témoignages et d'expériences ? Ces représentations non seulement interrogent mais remettent aussi en question la relation entre images, acteurs, espaces et contextes. Avec ces considérations théoriques pour toile de fond, l'enjeu est de penser à nouveaux frais le rôle que la tradition visuelle sportive a joué dans la définition et la fixation de normes liées à la sociabilité, à la pensée du corps et du temps, et aux rapports des groupes et des individus aux espaces et aux pouvoirs. Plus généralement, une telle étude questionne la place des images dans les recherches en sciences humaines : si elles ne sauraient être le reflet " objectif " d'une quelconque réalité, elles ont bien un rôle dans la construction des expériences et des normes.

\section{Continuités et ruptures}

Dès le $\mathrm{XV}^{\mathrm{e}}$ siècle, la figuration des jeux physiques réinvestit des motifs en partie déjà présents dans la culture visuelle médiévale. Les producteurs d'images renouvellent cet imaginaire à la lumière de la redéfinition prémoderne du jeu (ARCANGELI, 2003). D'une part, au XV siècle et au début du siècle suivant, les jeux (tournois, balle, paume...) continuent d'être, dans les représentations, autant d'indices d'un idéal social d'abord courtois, puis courtisan. Toutefois, on assiste à la fois à la diversification et à la multiplication 

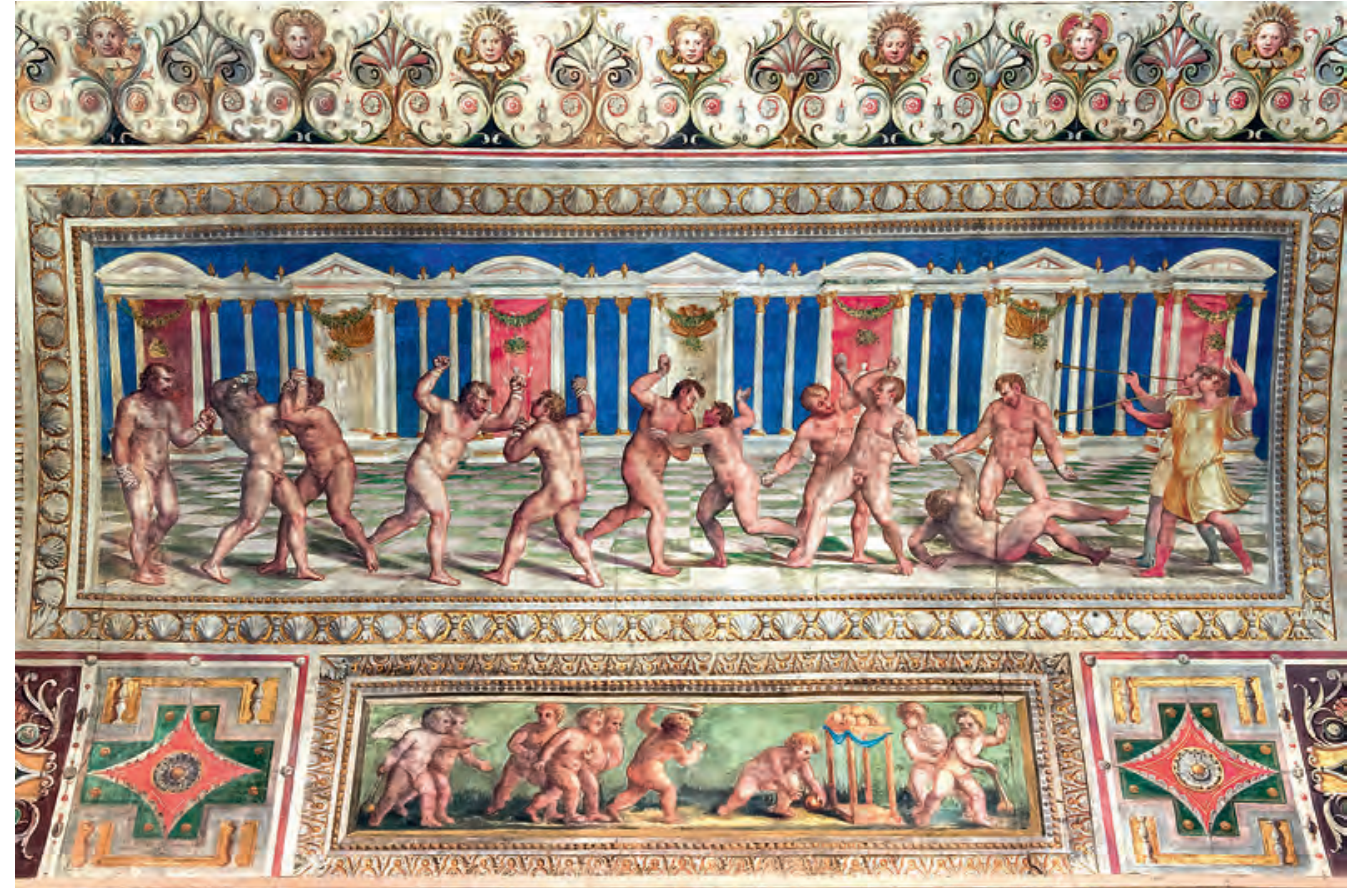

1. Leonardo Brescia, La lutte et la boxe, 1572-1574, Ferrare, castello Estense, sala dei Giochi.

de ces productions visuelles. Très tôt, en Italie centrale et septentrionale, elles ornent les salons des palais de l'aristocratie : à Milan, au palazzo Borromeo et dans la bicocca degli Arcimboldi, tout comme à Bracciano, dans le château des Odescalchi, des fresques monumentales mettent en scène la vie de la noblesse associant des jeux, tels que la palmata et la balle, aux occupations féminines. Dans la culture matérielle - coffres de mariage et plateaux d'accouchée d'Italie centrale ou encore livres d'heures enluminés dans le Nord de l'Europe - ce sont des jeux liés au calendrier festif et rituel qui sont mis en image, avec pour toile de fond la réalité urbaine davantage que l'idéal ludique courtois. Le peintre florentin Scheggia figure des jeunes gens jouant au civettino (une variante de la main-chaude) dans les rues de Florence (v. 1450, Florence, palazzo Davanzati) ; Giovanni di Francesco Toscani peint un palio sur la place San Pier Maggiore (1418, Cleveland Art Museum), tandis que dans les marges du Livre du Golf les enlumineurs de l'atelier de Simon Bening déclinent diverses activités sportives et ludiques de plein air (1520-1530, Londres, The British Library). Par ailleurs, ces scènes adoptent une tonalité politique et officielle lorsqu'elles sont intégrées aux lieux institutionnels et aux résidences princières : en témoignent les fresques du palio de Saint-Georges dans la salle des Mois du palais Schifanoia à Ferrare (1476-1484) et, différemment, celles du calcio que Jan Van der Straet peint au palazzo Vecchio de Florence vers 1560 (VAN SASSE VAN YSSELT, 1993).

D'autre part, la culture archéologique renaissante permet d'expliquer le recours à un répertoire formel et ludique antiquisant dans nombre de représentations proposant une revalorisation de la gymnastique de l'Antiquité. La lutte, la natation, le pugilat, les haltères ou le saut figurent dans les fresques de la salle des Jeux du château d'Este à Ferrare (1572-1574 ; fig. 1 ; LUCCHINI, 1987 ; CAPOROSSI, 2002) et dans les gravures exécutées par Cristoforo Coriolano pour illustrer le De Arte gymnastica de Girolamo Mercuriale (Venise, 1573 ; fig. 2 ; SIRAISI, 2003 ; GAGE, 2008 ; VAGENHEIM, 2008 et 2010). Or, cette valorisation des sports des Grecs et des Romains est déjà à l'œuvre au début du XV siècle dans les images des Enfants 
2. Girolamo Mercuriale, De Arte Gymnastica, Venise, 1573, livre II, fol. 112-113

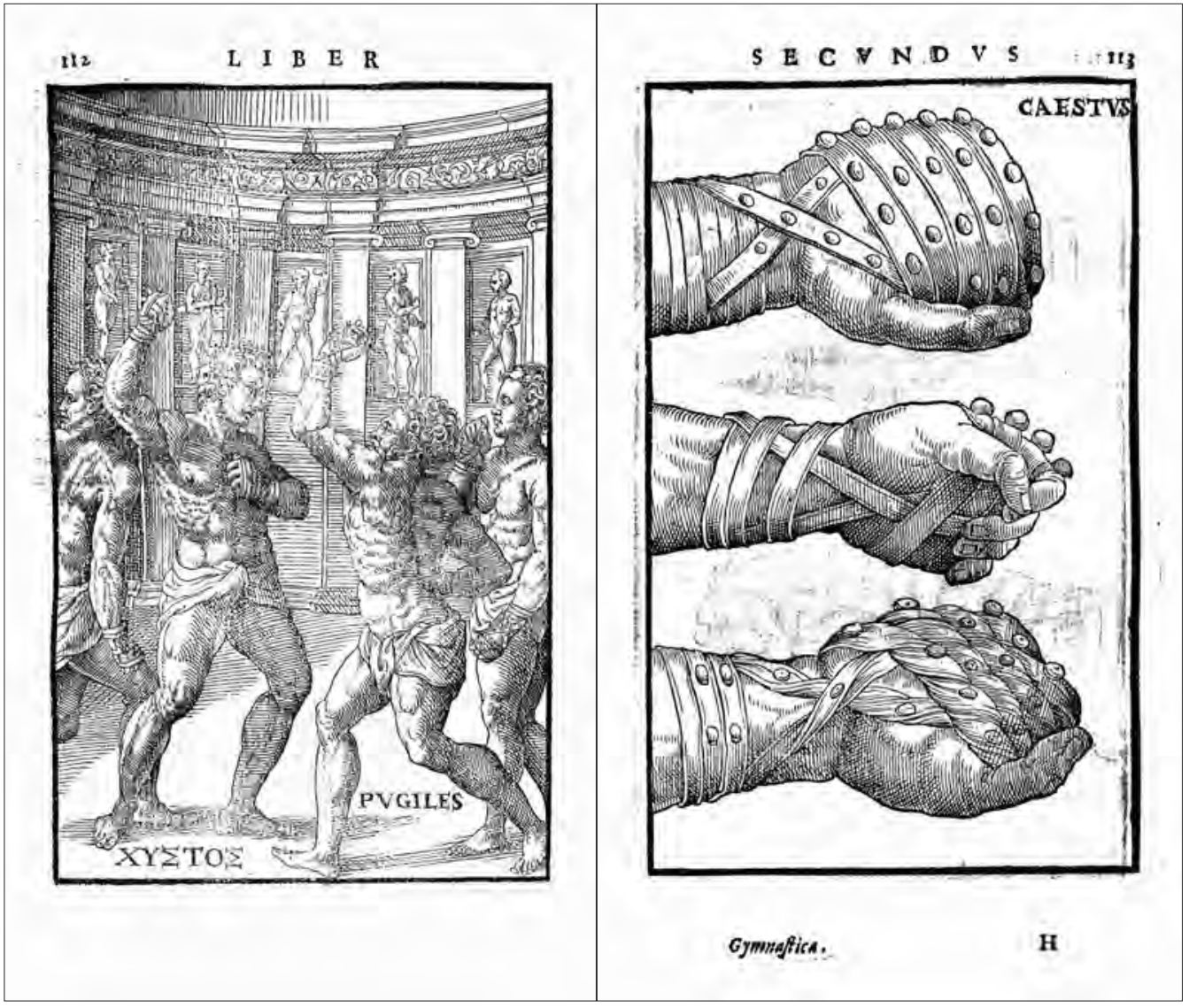

des planètes ${ }^{8}$, et plus particulièrement, dans celles figurant le Soleil (FENECH KROKE, à paraître). Les hommes nés sous l'influence de l'astre y sont présentés comme aptes à l'administration de l'État et de la justice, à la pratique religieuse et aussi aux activités athlétiques. C'est pourquoi ces représentations astrologiques montrent des dévots en prière, des rois en majesté et des gymnastes. Si l'iconographie apparaît dans les pays germaniques vers les années 1430, elle est fixée dans les enluminures, attribuées à Cristoforo de Predis, du De Sphaera de Francesco Sforza. Les scènes relatives à la dignité royale et à la dévotion occupent un folio autonome tandis que, juste sous la figure du Soleil (fig. 3), les gymnastes présentent une série d'exercices escrime, lutte, javelot, perche, haltères et saut acrobatique - ayant une connotation clairement antiquaire. On retrouve un dispositif analogue dans la gravure que Baccio Baldini exécute vers 1460 : l'iconographie septentrionale y est réélaborée dans une variante caractérisée par l'actualisation du contexte et la culture antiquaire dont relèvent les exercices et surtout le triomphe de la planète. Avant de disparaître complètement à la fin du XVI siècle, le modèle gymnique antiquisant et astrologique est porté à son ultime paroxysme par Jansz Müller qui fait des athlètes les uniques représentants des Enfants du Soleil.

Ce renouvellement de l'imaginaire ludique et du corps athlétique est servi par la multiplication et la reproductibilité des images comme par la diffusion des pratiques sportives à l'échelle européenne, grâce à une économie du jeu et à la mobilité de ses acteurs. Certains sports deviennent même de véritables attributs nationaux : dans la série allégorique gravée par Raphaël et Jan Sadeler (v. 1560), d'après des dessins de Hans Van Aachen, on trouve, 
aux pieds d'Italia, le ballon et le brassard de la balle au poing, Germania est figurée avec les épées de l'escrime tandis que, sans surprise, à la personnification de la France sont associées raquettes et balles du jeu de paume.

\section{Une Bildgenese du corps sportif}

De l'utilité des jeux sportifs pour la santé (ARCANGELI, 1999 et 2014) et des politiques éducatives (MERLOTTI, 2001 ; VAUCELLE, 2009b) témoignent les nombreux portraits d'enfants nobles avec raquettes et autres battoirs : ceux ayant pour modèle le jeune Federico Ubaldo della Rovere (par Barocci, Ridolfi et Vitali) ou encore les tableaux répertoriés dans les inventaires Barberini à Rome. Dans la France d'avant 1700, seuls le Portrait de Charles IX enfant (1552, Chantilly, musée Condé) par Germain Le Mannier et celui du jeune Louis XIV attribué à Philippe de Champaigne (v. 1650, Amboise, Fondation Saint-Louis) ${ }^{9}$ signalent l'intégration de l'exercice physique aux pratiques éducatives dans la préparation des jeunes princes au gouvernement de l'État. À l'apparition de ces portraits il faudrait également associer l'essor des " jeux d'enfants ", dont l'une des premières occurrences est la série de dessins d'inspiration antiquaire que Marco Zoppo exécute vers $1460^{10}$. Ce type de productions signale, entre autres choses, la place de l'exercice physique dans la pensée médico-philosophique - de Marsile Ficin (MORIN, 2009), par exemple - tout comme dans les nouvelles pédagogies annoncées par le $D e$ Ingenuisi moribus (1395) de Pier Paolo Vergerio, puis développées par le De Educatione liberorum (1443) de Maffeo Vegio, la Linguae latinae exercitatio (1538) de Juan de Vivès (RENSON, 1982) et par l'œuvre d'Érasme. Les préconisations de ces humanistes sont mises en pratique dans la $\mathrm{Ca}^{\prime}$ Iocosa ("Maison joueuse ") fondée à Mantoue dans les années 1420 par Vittorino da Feltre dans le but d'éduquer les enfants Gonzague (FREDDI, 2017). Parallèlement aux études, on y pratiquait jusqu'à l'âge de 10 ans les jeux de balle collectifs et la course, puis on y était initié à l'équitation, à la natation, à la lutte et à l'escrime. Ces auteurs intègrent le sport à l'éducation pour sa fonction pédagogique et hygiénique, anticipant la systématisation de ces politiques chez Jan Amos Comenius au XVII ${ }^{\mathrm{e}}$ siècle $^{11}$ et dont la culture visuelle témoigne déjà un siècle auparavant (VELDMAN, 2001 et 2004) ${ }^{12}$. Dès les années 1560, les éducateurs jésuites jouent un rôle dans ce changement ; ils redimensionnent la place de la mortification dans la formation des jeunes, en estimant plus efficace de fortifier, à travers l'exercice, non seulement le corps mais aussi l'esprit d'émulation, fondement du système éducatif jésuite. À la gymnastique des Antiques, à laquelle incitent les humanistes, on préfère les jeux contemporains, même ceux d'origine populaire - le ballon, la course, le mail ou encore les quilles (UlLmanN, 1965) : on le constate par exemple dans

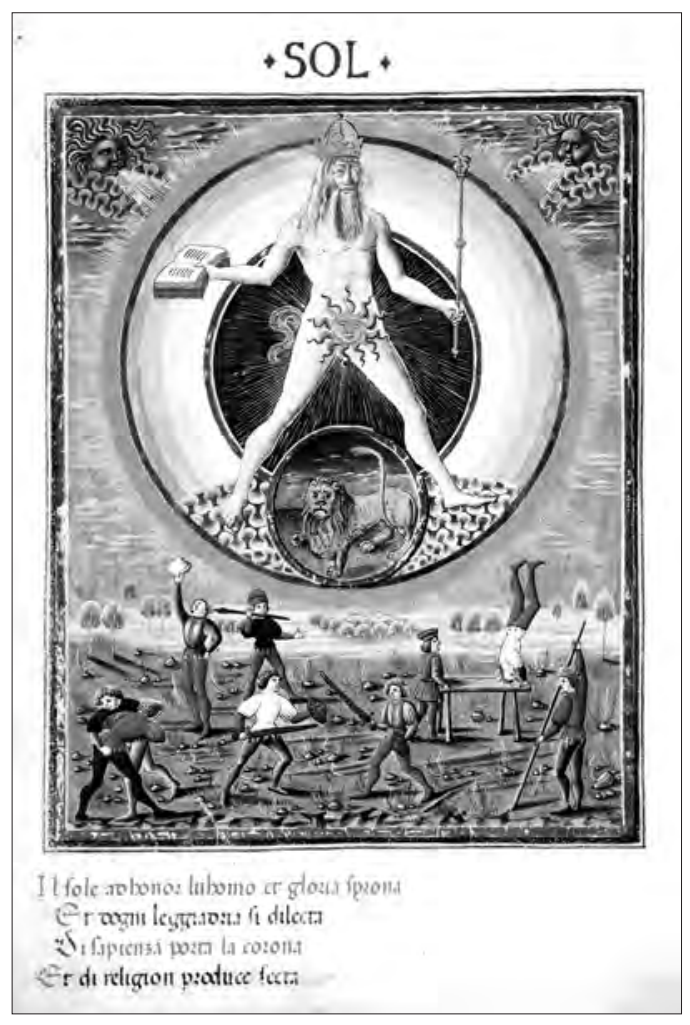

3. « Les Enfants du Soleil », dans Sphaerae coelestis et planetarum descriptio De Sphaera, vers 1450, Modène, Gallerie Estensi, Biblioteca Estense Universitaria. 


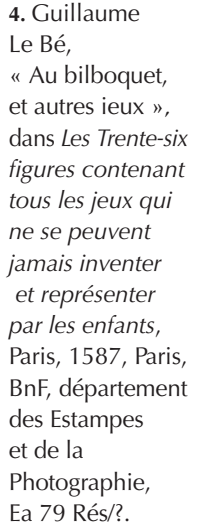

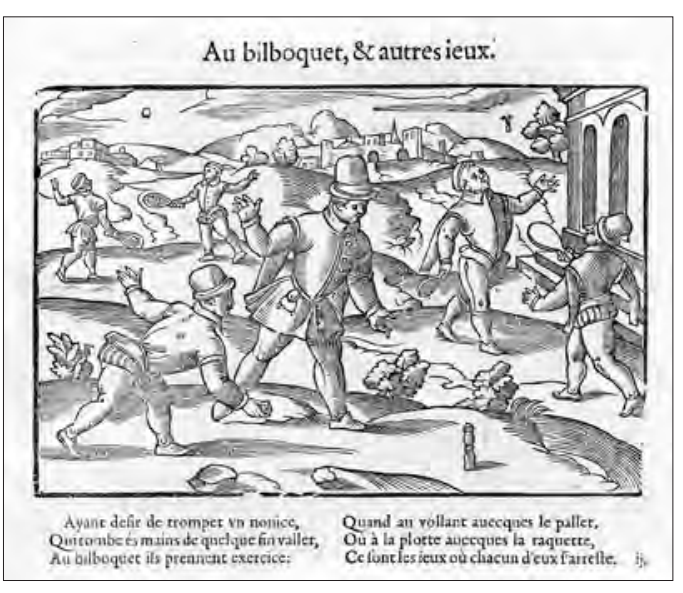

Les Trente-six figures contenant tous les jeux qui ne se peuvent jamais inventer et représenter par les enfants, un recueil de gravures imprimé à Paris vers 1587 par Guillaume Le Bé13 (MANson, 2003). Cette série d'estampes populaires organise les jeux en fonction des âges et en commente brièvement la pratique par le biais de vers. Si l'on y tient les jeux de la petite enfance comme nécessaires pour éveiller les tout-petits, lorsque l'on s'attarde sur les jeux d'exercice liés à un âge plus avancé, le graveur figure des enfants pris dans les mouvements et la gestuelle ludiques des quilles, du volant, de la longue et de la courte paume, de la balle, du tir à l'arc, ou encore du cerceau (fig. 4). Malgré leur pauvreté, certains vers mettent l'accent sur l'adresse corporelle, la vigueur, le " merveilleux effort " produit par l'exercice et insistent sur la question de l'échauffement nécessaire à l'activité physique de la jeunesse. Certes, la série constitue une taxinomie des jeux enfantins et s'inscrit dans une lignée qui va du Livre des Enfants $^{14}$ ou des dessins de Zoppo jusqu'aux séries gravées par Claudine Bouzonnet-Stella, Giacinto Gimignani ou par Louis Testelin. Mais, contrairement aux exemples antérieurs et postérieurs qui énumèrent les jeux d'enfants tout en s'inspirant des modèles antiques et renaissants du putto joueur, les Trente-six figures paraissent avoir d'autres finalités que celle taxinomique. En effet, elles rendent compte en filigrane des politiques éducatives et de la fonction sanitaire du sport à la fin du XVI ${ }^{\mathrm{e}}$ siècle.

En se fondant sur la notion galénique des complexions et sur les âges de la vie, les médecins de la Renaissance - Bartolomeo Platina, Ambroise Paré, Girolamo Mercuriale considèrent que l'enfance et l'adolescence sont les âges où le corps possède le plus de chaleur et d'humidité. L'échauffement engendré par l'exercice et l'assèchement qui s'en suit étaient tenus pour adaptés à ces âges puisqu'ils devaient accompagner, physiologiquement, l'évolution du corps vers le troisième âge, la jeunesse ou virilité " chaud $\delta$ seiche ". Antonio Scaino insiste aussi sur cette idée dans son Trattato del giuoco della palla (Venise, 1555), le premier traité de la modernité consacré aux jeux de balle :

Pendant l'enfance, l'exercice fait sans ordre et mesure, pourvu qu'il ne soit pas trop fatigant, est bon pour digérer une telle abondance d'humeurs typique de cet âge et pour mieux attiser la chaleur qui y est moins vivace. Pendant la jeunesse, l'âge se faisant plus robuste en raison de la chaleur plus active et véhémente, il convient que l'exercice soit encore plus robuste mais davantage réglé et mesuré dans le temps que celui pratiqué dans l'enfance. On peut réserver à l'âge viril le genre d'exercice de la jeunesse mais avec davantage de mesure et de modération puisqu'il manque (à cet âge) cette abondance $\mathrm{d}^{\prime}$ humeurs dont regorgeaient la jeunesse et plus encore l'enfance ${ }^{15}$.

La modération dans l'exercice se doit d'augmenter avec l'âge en fonction de l'évolution du corps, idée réitérée dans Trois dialogues de l'art de sauter et voltiger (Paris, 1599) d'Arcangelo Tuccaro, le premier manuel de saut acrobatique (SCHMIDT, 2008 et 2009).

L'interrelation entre culture visuelle, culture médicale et pédagogie donne sens à l'articulation entre physiologie et convenance dans la construction du corps sportif (qui est également un corps social et politique), mais elle permet aussi de mieux comprendre les dispositifs 
de figuration distinguant le jeune joueur de l'adulte. Si les artistes s'attachent en effet à représenter le mouvement dans les jeux d'enfance, en revanche lorsqu'ils figurent l'âge viril le traitement change sensiblement : le sportif noble est un joueur statique ! La fonction hygiénico-médicale et éducative cède alors la place à la fonction sociopolitique du sport (GUERZONI, 1995 et 1996 ; MCCLELLAND, 2003). Cela est évident dans le cas des jeux de balle. Peint vers 1510, le Portrait de joueur de scanno par Francesco Beccaruzzi (fig. 5) est un témoignage rare de ce jeu et d'un

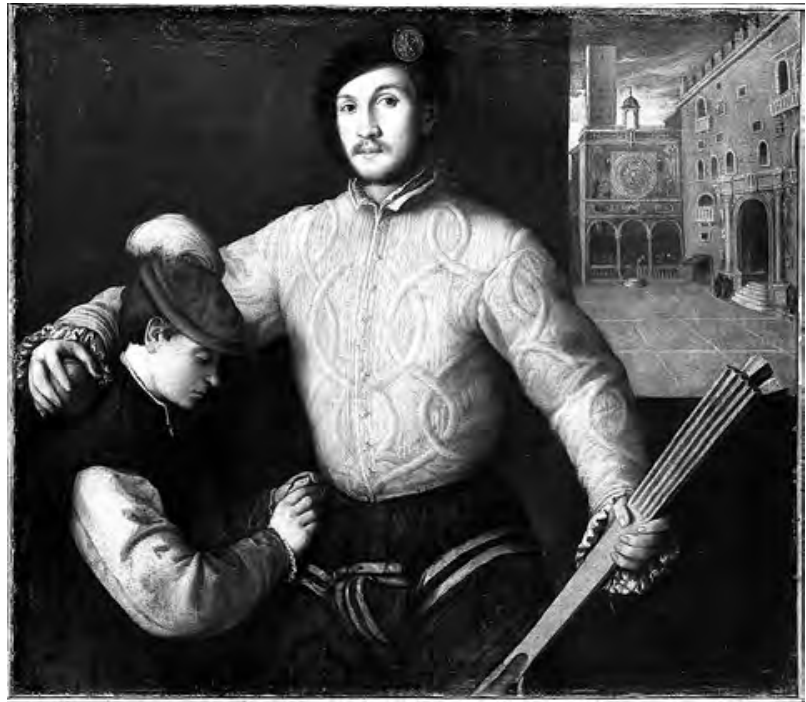

5. Francesco Beccaruzzi (attr.), Portrait de joueur de palla da scanno avec un page, v. 1520 , huile sur toile, Berlin, Staatliche Museen, Gemäldegalerie, inv. $\mathrm{n}^{\circ} 158$. portrait de "sportif " adulte (FENECH KROKE, 2014). La petite balle en cuir et le battoir présentés ostensiblement, la gestuelle et l'expressivité des figures, leur mise en scène à l'avant-plan de la piazza dei Signori à Trévise, l'action du page, magnifient l'homme et idéalisent le scanno comme loisir aristocratique. L'association figurale jeu/effigie révèle ainsi les implications sociopolitiques et économiques de ce type de jeux de balle. Si au Moyen Âge ils étaient associés aux passe-temps féminins, la première modernité en fait des activités masculines; si au Moyen Âge, ils se déroulaient dans le jardin clos (allégorisé), dès le $\mathrm{XVI}^{\mathrm{e}}$ siècle on ne joue plus uniquement dans le jardin ou dans la cour des palais. La balle et le ballon deviennent des pratiques spectaculaires d'ostentation pour les élites. "Le jeu de balle doit se faire presque toujours en public ; et c'est un de ces spectacles auxquels la multitude apporte beaucoup d'ornement " écrit Baldassarre Castiglione dans Le Livre du courtisan (1528; II, 10). En Angleterre, Thomas Elyot (The Boke Named the Gouvernour, 1531) ou, dans un autre registre, William Shakespeare (As you like it, 1599) témoignent de cette nécessité pour le gentilhomme de démontrer, par ses compétences athlétiques, sa supériorité physique (et sociale) par rapport à l'homme simple. Le sport devient l'attribut de la noblesse (WILLIAM, 2008 ; FONTAINE, 2009). Suscitant une forme d'auto-contemplation collective, cette extériorisation des jeux conduit à distinguer les " loisirs d'ostentation " à la fonction politique, des "loisirs ordinaires " pratiqués dans le cercle social d'appartenance (ROMANI, 1995). Ainsi le portrait de Beccaruzzi célèbre-t-il les valeurs du corps afin de faire du joueur trévisan un paradigme de virilité et de noblesse : la pose, le regard sur l'observateur, la monstration du scanno le manche appuyé sur la hanche - tel un bâton de commandement - réélaborent les marqueurs du portrait militaire contemporain (pensons au Portrait d'un homme d'armes, v. 1510, par Francesco Granacci). Tout comme la présence du page qui, noble lui-même, suggère que le jeu n'est pas un loisir impromptu mais bien un spectacle politique. Par ailleurs, le page renvoie à la figure de l'écuyer au service du condottiere (le Portrait d'un capitaine avec deux pages de Paris Bordone ou le Portrait de Scipione Clusone par Tintoret), et signale par là même l'isomorphisme des jeux, de la politique et de la guerre (MCCLELLAND, 2003). Au Moyen Âge, joutes et tournois préparaient les corps à une guerre qui, par la nature de l'armement et des combats, requérait puissance et force. Or, la caractérisation prémoderne du corps et des jeux sportifs est à mettre en relation avec le renouvellement des techniques militaires, l'introduction des armes à feu et l'institution 


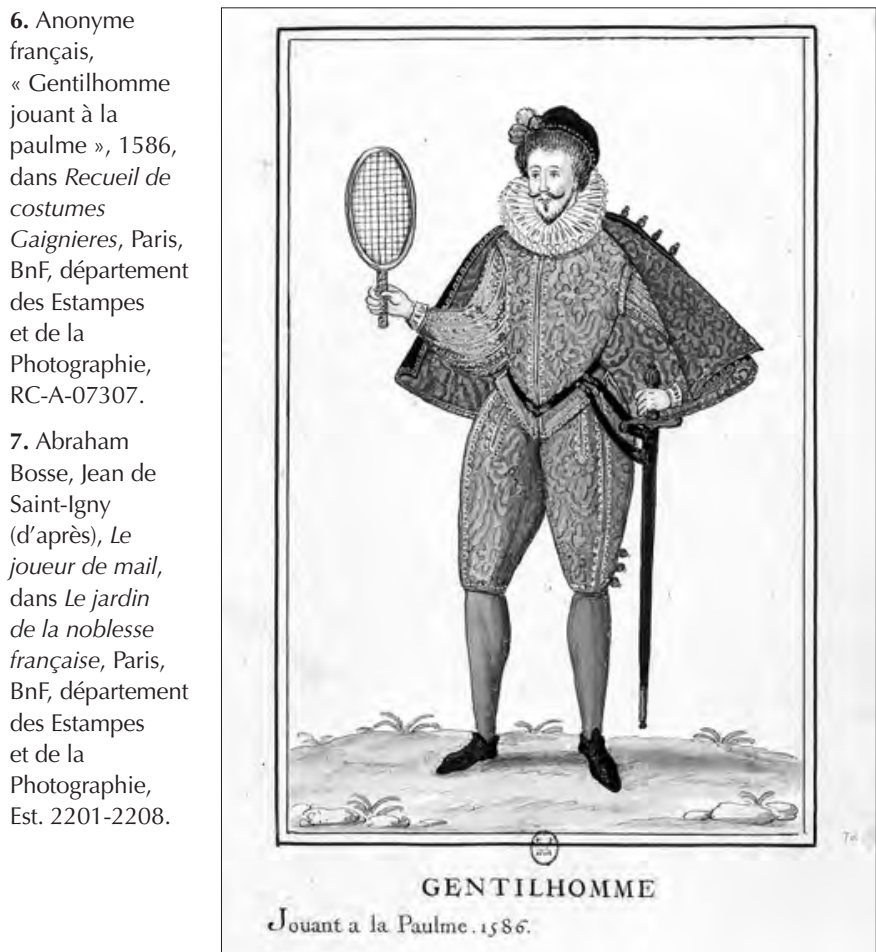

d'armées régulières. L'adresse physique davantage que la force, l'expertise stratégique et l'agilité deviennent les indicateurs du nouvel art militaire (TURCOT, 2016). Considérant les normes des jeux de balle comme une transposition des lois qui règlent le monde, Scaino va jusqu'à s'approprier les thèmes de la littérature politique et fait de ces pratiques une propédeutique à l'action politique, redessinant les marges sérieuses du jeu ${ }^{16}$.

Toutefois, c'est la culture visuelle française des années 1580 qui produit, dans un livre de costumes, l'un des premiers modèles de sportif noble (fig. 6) : il s'agit de deux estampes presque identiques qui présentent un "Joueur de courte paulme " (1581) et un "Gentilhomme jouant à la paulme ${ }^{17}$ " (1586). Or, la figure y est montrée en position frontale et statique, et seules la raquette et les inscriptions le qualifient comme joueur tandis que les vêtements signalent sa noblesse. La posture et la neutralité du fond écartent toute allusion à l'espace et à l'action ludiques. L'autre élément indiciel qui fait pendant à la raquette est l'épée, signalant l'interrelation entre la paume et l'escrime : si jusqu'au début du XVI ${ }^{\mathrm{e}}$ siècle, le noble est ceint d'une épée dans les portraits militaires, plus tard c'est la rapière, en tant qu'arme civile, qui devient un marqueur social. En effet son port n'était autorisé qu'aux nobles en vertu du droit à l'autodéfense, mais interdit aux laquais, aux écoliers et aux clercs (BRIOIST, DRÉVILLON, SERNA, 2002 ; VAUCELLE, 2009a). Signes de l'honneur aristocratique et bourgeois, l'épée et la raquette sont ici les attributs de deux pratiques appréciées parce qu'elles imposaient maintien, mesure et contrôle des mouvements du corps. Plus de quarante ans plus tard, c'est une gravure de mode tirée du Jardin de la Noblesse françoise par Abraham Bosse qui témoigne de l'évolution des comportements ludiques (fig. 7). Ceint de la rapière, coiffé d'un large chapeau à plume, le club de mail renversé, le joueur noble prend la pose avec une nonchalance ostentatoire, représenté de trois quarts et légèrement tourné vers le spectateur. Bosse en fait une figure monumentale et, à nouveau, statique. L'action physique est omise au profit d'une image encodée du corps " sportif ». Les sources signalent l'évolution de la gestuelle ludique : au nom de la bienséance, 
le joueur est contraint à un contrôle accru de ses mouvements dans une société policée qui dicte les règles très strictes du maintien. Cela entraine une baisse d'intérêt pour les jeux physiques emportés, comme la paume ou le ballon, au profit de ceux qui requièrent plutôt prestance et élégance (BELMAS, 2006 et 2011 ; VIGARELLO, 1988, 2002 et 2005). L'allure du joueur de Bosse cristallise ce changement et l'engouement pour le mail par lequel s'exprime la bienséance dans les attitudes, le mouvement et la tenue vestimentaire. L'action se dérobe au regard : à l'arrière-plan, trois silhouettes jouent dans un parc. Dans l'élaboration progressive de ce corps sportif idéalisé, le joueur est donc principalement un corps social et politique ${ }^{18}$ et paradoxalement un corps statique. L'action est mise à distance au profit de l'image du corps sportif comme artefact de civilisation.

\section{Le geste et la règle. Technique du corps et tékhnê ludique}

À ce processus d'idéalisation de l'homo sportivus prémoderne fait écho la normalisation des techniques ludiques et corporelles décrites dans une littérature dédiée (BASCETTA, 1978) dont l'essor se met en branle à partir du Xve siècle. Dans ces traités des jeux les images donnent à voir les contenus textuels tout en façonnant une nouvelle norme ludique. Ce sont les textes consacrés à l'escrime comme pratique sportive qui ont exploité, les premiers, le potentiel des images (CASTAGNARO, 2009-2010; ANGLO, 2010). Dans les années 1410, le manuscrit du De Arte luctandi de Fiore dei Liberi mêle instructions techniques et représentations des positions de l'escrimeur (Paris, BnF, MS Latin 11269). Avec le passage au livre imprimé, cette tendance se cristallise : l'Opera Nova dell'arte delle armi (Modène, 1536) d'Achille Marozzo paraît en effet avec un appareil iconographique très conséquent. Si, dans la première édition, les quatre-vingt-trois xylographies n'ont qu'une efficace réduite dans la transcription des gestes (à cause de la technique, des costumes complexes des escrimeurs, de la réutilisation de planches), l'édition de 1568, avec ses gravures sur cuivre, présente des dispositifs visuels bien plus performants. Le Trattato di Scientia d'armi (Rome, 1553) par Camillo Agrippa est symptomatique du rôle donné aux images dans la fixation des techniques : non seulement ses gravures présentent les mouvements sur la base de schémas géométriques et les escrimeurs sont maintenant figurés nus afin de décrire avec précision les gestes mais aussi, pour la première fois, on figure diverses positions dans une même page dans le but de visualiser tout le déploiement des mouvements. Une posture est parfois montrée de face, de profil et quelquefois de trois-quarts, tandis que des lignes matérialisent les déplacements de l'épée dans l'espace. Plus intéressant peut-être, dans les gravures de la deuxième partie (chap. X-XIII) quatre figures prises dans un combat structurent la composition : en réalité, il s'agit d'un seul couple d'escrimeurs, dédoublé afin de présenter une position en début et en fin d'attaque. Ce choix n'est pas tant dicté par un souci d'économie que pour figurer les mouvements dans le temps et dans l'espace (fig. 8). Il en est de même dans le traité sur la voltige de Tuccaro, dont les gravures sont caractérisées par deux types de dispositifs : selon le premier, le saltatore isolé est

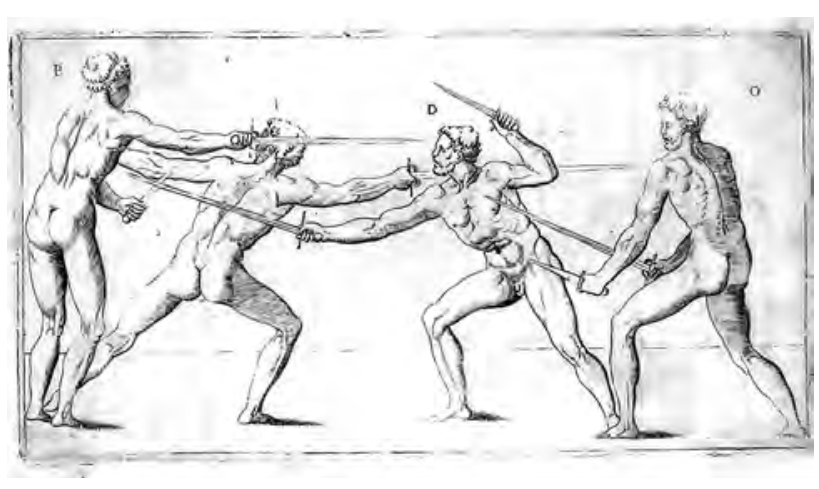

8. Camillo Agrippa, Trattato di scientia d'armi, Rome, 1553, Seconda parte, XIII. 
figuré dans le moment initial d'un saut puis, dans une seconde gravure, dans la phase intermédiaire ou finale. L'insertion de ces images se fait en fonction de la description du mouvement dans le texte. Dans le second type de gravure, le voltigeur est présenté, sur une seule planche, à des moments différents d'un même exercice (fig. 9).

Il faut faire deux remarques à propos de cette littérature et de son appareil iconographique. D'abord, la pratique sportive y est l'objet d'un détournement : accompagnés de dispositifs visuels qui génèrent aussi l'essor d'une nouvelle tékhnê ludique, ces traités participent du façonnage de l'imaginaire du corps sportif moderne, qui est un corps " performant " physiquement mais aussi socialement. En effet, si l'on s'en tient aux exemples cités, l'escrime s'affranchit de l'art de la guerre tandis que le saut acrobatique est dissocié des pratiques du jongleur, figure à la marge des sociétés médiévales et prémodernes. Ensuite, les postures, les mouvements des bras et des mains, le positionnement des pieds, le maniement des instruments sont consignés dans les images de manière à ce que le lecteur, se détournant du texte, puisse saisir (intuitivement) des techniques du corps. Certes, l'apprentissage de celles-ci ne pouvait être effectif qu'après de longues heures de pratique, de répétitions méthodiques visant à atteindre la performativité des automatismes, des savoir-faire incorporés (DESCOLA, 2017). Mais la différence entre le fait de lire comment placer son corps et le fait de le voir, ne serait-ce qu'en image, est de taille ! Les savoir-faire corporels sont en effet difficilement objectivables par le langage, quant à lui toujours construit en séquences de phrases. Soumise à l'analyse, l'action ludique doit être déconstruite, reconstruite et modélisée. Et les images, assurant une agentivité dans le décryptage des techniques ludico-corporelles, constituent dès lors un palier spécifique dans le processus d'apprentissage des gestes par le corps du lecteurspectateur. En cherchant à saisir et à mesurer le mouvement en image, celle-ci le décompose dans une abstraction théorique qui, paradoxalement, l'arrête ${ }^{19}$.

En somme, les dispositifs visuels de cette littérature sportive permettent, d'une part, de dissocier les jeux physiques des pratiques auxquelles ils étaient associés (la guerre ou le spectacle); d'autre part, par la décomposition analytique et la visibilité instantanée auxquels ils donnent lieu, d'analyser et de saisir les mouvements, les déplacements, les enchaînements de

9. Arcangelo Tuccaro, Trois dialogues de l'exercice de sauter et voltiger en l'air..., Paris, Claude de Monstr'œil 1599, p. 142

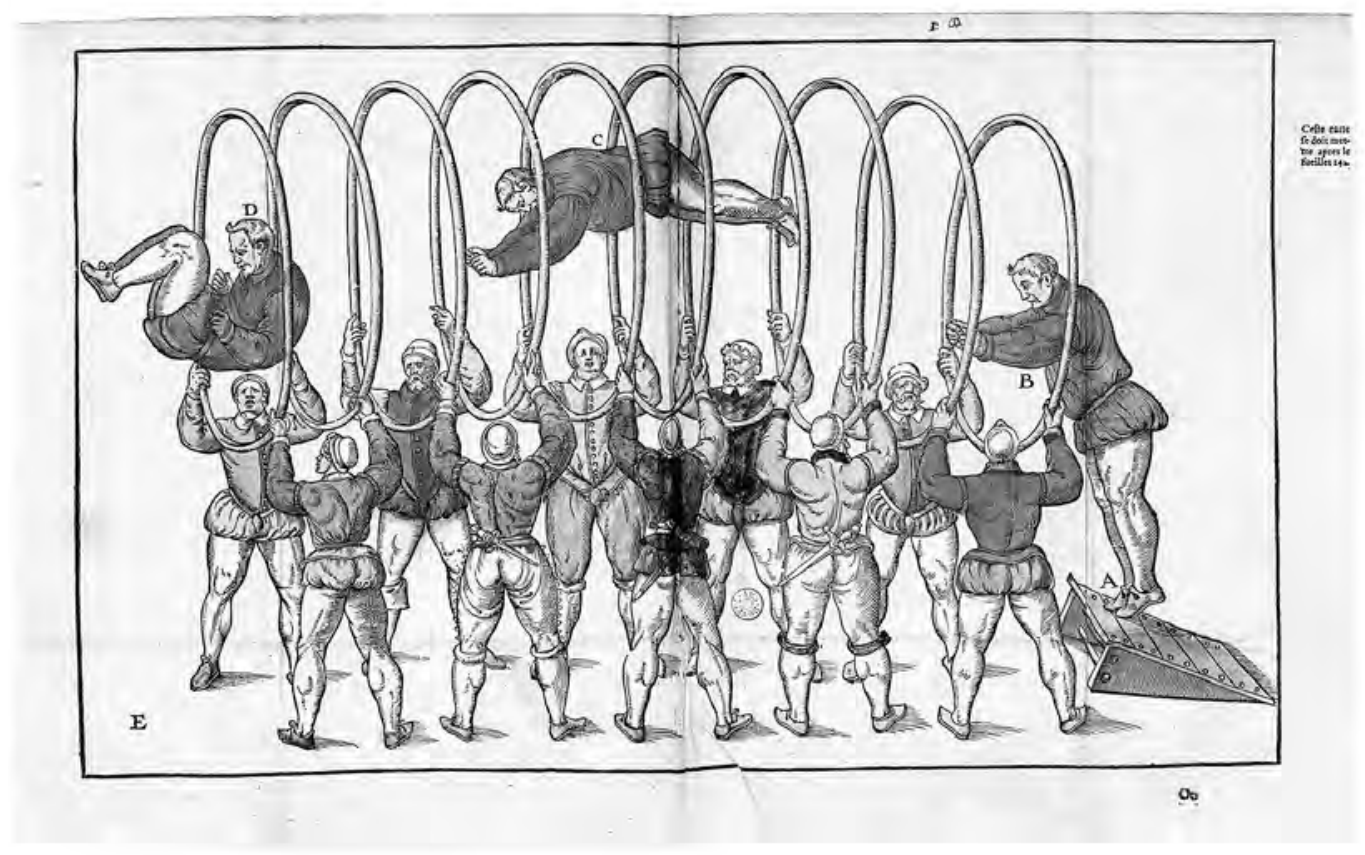


gestes dans l'espace et aussi dans le temps. On exploite les potentialités de l'image pour répondre aux exigences d'une littérature sportive naissante et de ses lecteurs. Nouveaux moyens techniques de visualisation des mouvements et sports ont toujours fait bon ménage : sans aller jusqu'aux modélisations 3D que permet la technologie actuelle, pensons à la photographie qui, au XIX ${ }^{\mathrm{e}}$ siècle, a permis d'étudier les mouvements d'animaux et d'humains, ces der-

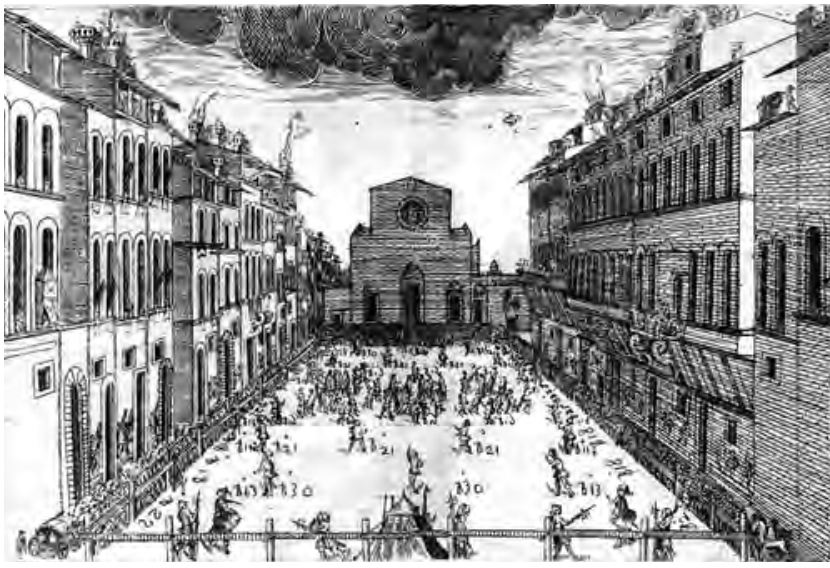

10. Giovann de' Bardi, Discorso sopra' giuoco del calcio, Florence, (1580) 1615. niers parfois en train de pratiquer un sport. Or, contrairement aux chronophotographies et aux graphiques de Marey et aux planches de Muybridge qui analysent une action en autant d'instants " quelconques " qu'il y a de prises de vue, les gravures des traités prémodernes n'arrêtent qu'un nombre très limité d'instants aptes à synthétiser, intuitivement, le déroulement d'un geste (TORTAJADA, 2008).

D'autres préoccupations émergent des six illustrations du Trattato dei giuochi della palla de Scaino. L'ouvrage examine, distingue et compare les jeux de balle contribuant à la fixation, en Europe, des spécificités du ballon, de la palla al bracciale (balle au poing), du mail, de la paume et aussi, on l'a dit, de la palla da scanno. Accompagnant les explications (II, 11-12) sur la nature du cuir pour confectionner les balles, sur leur gonflement, leur poids et leur diamètre, les deux premières gravures présentent les instruments de la palla al bracciale et de la palla da scanno : on peut y voir les balles, les pompes à gonfler et les battoirs dont on indique les dimensions, les finitions et les rainures. Les quatre autres illustrations sont consacrées, quant à elles, aux espaces de jeu : le premier est un relevé du jeu de paume du château du Louvre adossé à l'enceinte de Philippe Auguste, tandis que les trois autres sont des plans types pour des terrains de courte paume, de gioco di paletta (raquette en bois), de paume avec la main et de tambourin. La codification des espaces ludiques est aussi à l'œuvre dans le Discorso sopra 'I giuoco del calcio (Florence, 1580) de Giovanni de' Bardi, dont l'unique gravure montre une partie sur la place Santa Croce, lieu traditionnellement destiné à ce sport (entre autres pratiques ludiques et spectaculaires). Sur le terrain, deux équipes de vingt-sept joueurs sont représentés en mouvement devant des spectateurs placés derrière des barrières. L'intégration dans l'image d'un système alphanumérique en fait moins un dispositif de type narratif qu'un modèle schématisant les rôles et les emplacements des joueurs dans une composition qui reste descriptivo-naturaliste (fig. 10). Toutefois, dans la réédition du traité datant de 1673, puis dans les Memorie del calcio fiorentino (1688) de Pietro Bini, une variante de cette composition présente les joueurs statiques dans la disposition " théorique " initiale. Dans une seconde illustration, ce dispositif devient un schéma légendé donnant les dimensions règlementaires du terrain, l'emplacement et le rôle des joueurs (BREDEKAMP, 1993).

Les images de ces techniques ludiques jouent la visualité et instaurent un régime de communication qui accompagne et/ou remplace celui du texte. De ce fait elles sont l'expression d'une normativité qui ne peut pas être simplement comprise comme la traduction visuelle des règles d'un jeu. Elles ne transmettent pas seulement des informations et des savoirs mais sont performantes du fait qu'elles produisent des normes relatives aux pratiques corporelles, sociales dans la vie individuelle et collective. 


\section{Allégorisations sportives}

$\mathrm{Au}$ vu de ce processus de normalisation par l'image du corps sportif, des techniques et des espaces, on pourrait croire que la charge édifiante qui caractérise dès le Moyen Âge les images des jeux s'atrophie. Or, cette composante allégorique peut aussi se greffer dans la stratification du sens de ces représentations.

La culture littéraire et visuelle de la Renaissance situe dans l'espace du jardin ces jeux pratiqués par la noblesse, les reliant ainsi à la tradition du locus amonus dérivé à la fois du mythe de l'Éden vétérotestamentaire et de celui de l'âge d'or ovidien que l'imaginaire renaissant redécouvre et revisite. Ces allusions à l'espace-temps idéalisé et improductif du jeu qui fait écho à celui des récits édéniques ou aux fables mythologiques du bonheur originel connote positivement la nature des pratiques ludiques nobles. Dans cet état d'otium ludique, les hommes et les femmes profitent des joies d'un statut social, économique, politique et intellectuel qui les affranchit d'une utilisation du temps économiquement profitable. Les représentations des villas extra-urbaines avec leurs jardins sont le lieu de cette articulation entre jeu et oisiveté édénique : dans la gravure de la villa d'Este à Tivoli qu'Étienne Dupérac publie en 1573, tout comme dans la Villa Lappeggi peinte par Juste Utens en 1599, les pratiques ludiques aristocratiques sont les seules activités humaines représentées : dans la première, six figures jouent à la paume dans un espace dédié que l'inscription nomme "giuoco di palla ". Dans la toile florentine, c'est devant la villa qu'un groupe d'hommes joue à la balle au poing, alors que d'autres jouent au mail devant l'entrée pendant que trois personnages reviennent de la chasse. Dans la partie droite de la bâtisse, on reconnaît aussi la structure d'un jeu de paume.

Ces dispositifs sont tout aussi fréquents dans la culture visuelle septentrionale, où l'on joue dans les parcs et les espaces périurbains. Toutefois, dans ces productions la portée allégorique est d'une autre nature. Herri Met de Bles place une partie de paume au centre de son Paysage avec David et Bethsabée (1535/1540 ; fig. 11), une ouvre qui a donné lieu à une dizaine de copies libres, certaines du pinceau de Lucas Gassel. L'insertion du motif ludique

11. Herri Met de Bles, Paysage avec David et Bethsabée, v. 1535-1540, Boston, Isabella Stewart Gardner Museum.

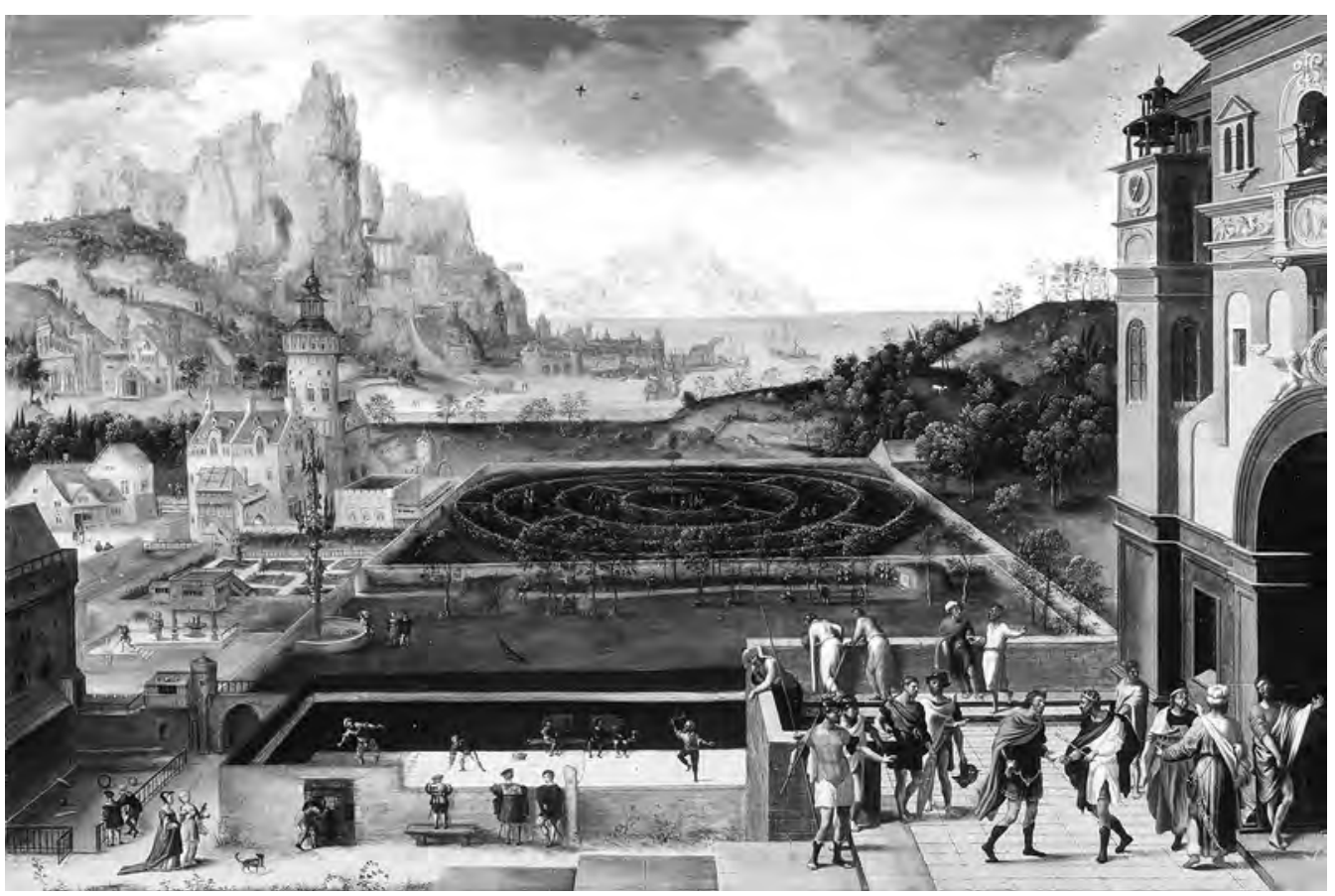




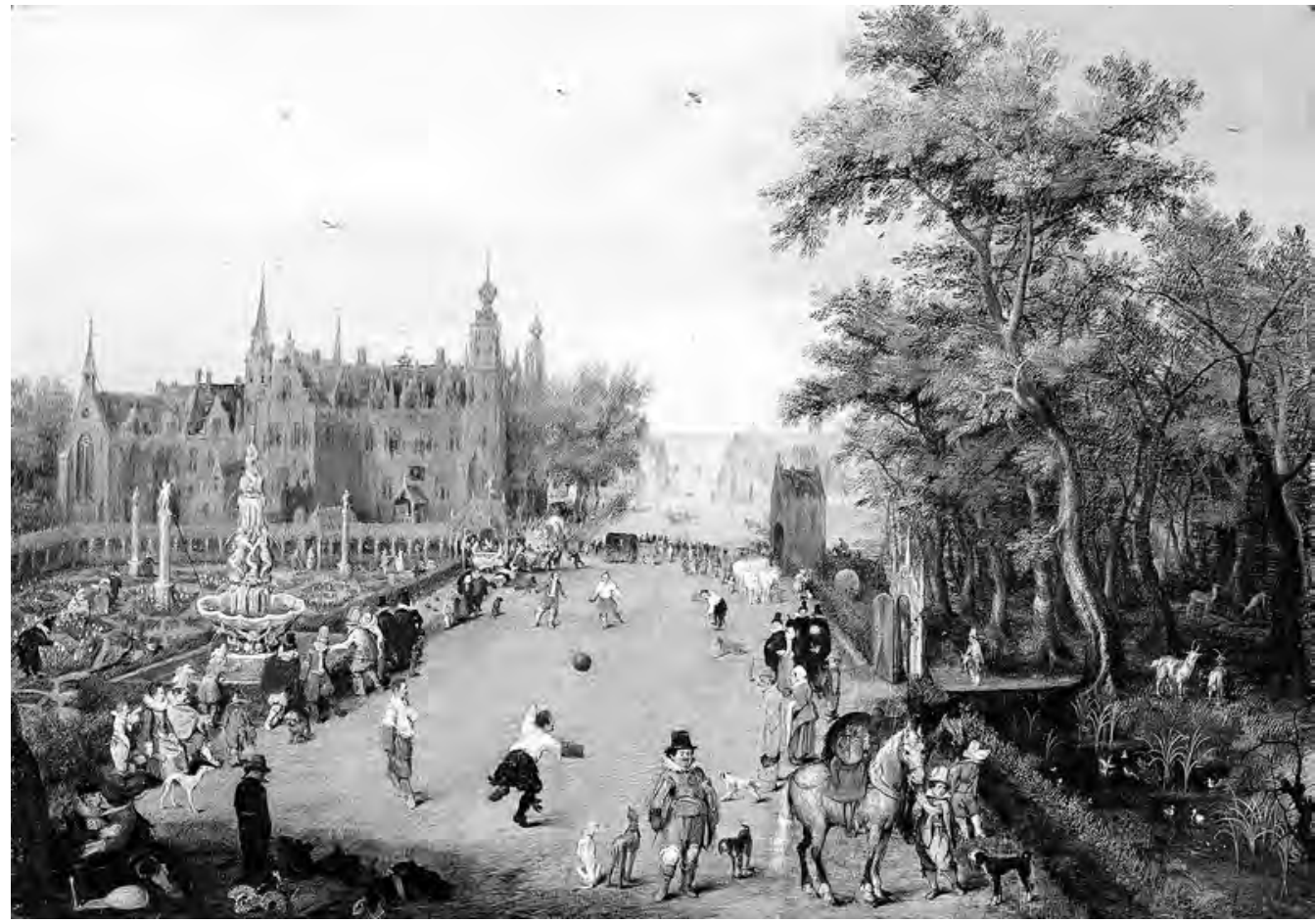

12. Adriaen Van de Venne, Partie de balle au poing, 1614, Los Angeles, The J. Paul Getty Museum.

rend compte de la portée symbolique et philosophique de la représentation (WEEMANS, 2002, 2012 et 2013, p. 135-147). Quelle relation peut-on établir entre une partie de jeu de paume et un roi adultère ? L'artiste n'illustre pas simplement l'épisode biblique mais en fournit une interprétation en inventant des procédés visuels où le jeu possède un rôle exégétique central. Dans le complexe réseau métatextuel qui façonne le sens de l'œuvre, l'image du parc et de ses espaces ludiques réélabore visuellement l'abbaye de Thélème telle que Rabelais l'a décrite (Gargantua, LV) : le verger, le jardin, la fontaine, le parc aux animaux sauvages et surtout les nombreux jeux (paume, balle, tir à l'arc, boules). Dominant toute la composition, la partie de jeu de paume devient une métaphore du regard et de la vision (en tant qu'expérience physique et spirituelle) du fait même que le jeu requiert une acuité visuelle exceptionnelle et que la présence de spectateurs activement engagés dans la partie fait allusion à l'acte de " voir ». Cette métaphore est en effet une constante dans la littérature de l'époque lorsqu'il est question du jeu de paume. Le tableau de Bles prolonge la tradition contemporaine des jeux de paume emblématiques : les deux emblèmes du Théatre des bons engins (V et XVL, 1539) de Guillaume de La Perrière, l'emblème 75 du Picta poësis (1552) de Barthelemy Aneau, ou celui des Emblemata (emblème 114, 1564) de Sambucus (BONDT, 2006, p. 193-197).

De même, nombreux sont les emblèmes dont le sens est porté par d'autres jeux sportifs : dans l'une des gravures de l'Academia sive speculum vitae scolasticae de Crispin de Passe, une partie de balle au poing décrit la vie estudiantine mais est aussi l'occasion d'une incitation à la modération (VELDMAN, 2002, p. 34-52). À la marge de la composition, en effet, le tas de vêtements dont les joueurs se sont délestés signale la métamorphose du corps social en corps hédoniste et le glissement de l'ostentation des signes vestimentaires vers l'ostentation d'un corps sportif. Indice d'ordre narratif, le tas d'habits est surtout le signe de l'abandon d'un " habitus " au sens maussien du terme. On le retrouve dans une miniature d'Adriaen Van de Venne (fig. 12), où deux équipes de trois joueurs s'affrontent dans un parc. L'un vient 
d'envoyer la balle vers celui qui, dans la diagonale, se tient prêt à la relancer pendant que son coéquipier l'observe attentivement. La partie se déroule devant un public où des nobles, des bourgeois, des marginaux urbains et des paysans se côtoient. L'attention portée à la mixité sociale et aux corps dynamisés, si elle relève d'un registre naturaliste, ne donne pas une image moins politiquement orientée du jeu pour autant, d'où émerge une vision de l'harmonie et de la paix politique et sociale des États de Hollande. L'image est néanmoins riche d'indices pointant aussi la nature criminogène que ce temps libéré et cet hédonisme ludique peuvent engendrer. Sur le terrain manque l'un des joueurs ; encore habillé et coiffé de son chapeau, celui-ci se tient, le brassard au bras, à l'écart, près de la fontaine, et en conversation avec un personnage dont l'accoutrement correspond exactement à celui de la personnification de la Fraude. Cet échange équivoque renvoie à la nature criminogène du jeu dans l'espace public : contrairement aux jeux de hasard, les pratiques sportives n'étaient pas condamnées per se, mais elles étaient perçues avec méfiance en raison des violences et des paris d'argent que les compétitions occasionnaient, un problème auquel les pouvoirs n'ont eu de cesse de se mesurer (CROUZET-PAVAN, 1993 ; ORTALLI, 1997 et 1999; WALKER, 1999; TADDEI, 2001 ; BELMAS, 2006 ; RIZZI, 2006 ; SCOTT BAKER, 2016). La présence du couple engagé dans les ébats amoureux à l'avant-plan du tableau révèle une autre utilisation métaphorique des jeux sportifs : l'association du corps ludique au corps érotique (MCCLELLAND, 2002). L'homme mordille l'oreille de la femme dont l'appétit sexuel est signifié par le luth retourné ; l'instrument est une métaphore érotique dans les images et dans la littérature en tant que son manche est une allusion au phallus en même temps que le terme néerlandais luit ( "luth ") évoque le sexe féminin (ZECHER, 2000). Ces mêmes allusions prennent des formes bien plus techniques dans une miniature de Hans Bol (1589, Gemäldegalerie, Berlin) tout comme dans une gravure de l'atelier de Crispijn de Passe (fig. 13) où l'image du gonflage de la balle devient la métaphore visuelle de l'acte sexuel, auquel font allusion les vers : " Ecce tumescentem follem quae caussa tumoris? Cum spiritu infusus liquor. " La connotation érotique de l'image est explicitée par les vers qui l'accompagnent dans une version de l'Énigme joyeuse pour les bons esprits : "Ie le vy squ'il tenoit entre ces deux genoux quelqu'une bien parée et passablement belle laquelle il l'embrassoit d'une manière telle qu'il luy faisoit monstrer son trou à tout le coup. Un autre ce pendant qui le voit, que sans poux, sans haleine, \& sans vent, sans cesse elle chancelle, s'approche, $\delta$ se choquant brusquement avec elle faict qu'il tient le dessus, $\delta$ elle le dessoubs. Après entre ses mains un long chose il empoigne qu'il luy met dans le ventre, $\delta$ hastant la besongne, pousse tant et souvent que grosse elle devint ${ }^{20}$."

La charge allégorique des activités sportives devient explicitement politique dans quelques-uns des dessins de l'album d'Adriaen Van de Venne (1625-1626, The British Museum ; ROYALTON-KISCH, 1988), offert probablement au Stadhouder Frédéric-Henri d'Orange par le roi de Bohème Frédéric V. Couronné en 1619, ce dernier fut destitué par Ferdinand II et, contraint à exil, il se réfugia à La Haye. Frédéric-Henri et Frédéric V s'étaient retrouvés pris entre deux conflits, la longue guerre de Quatre-vingt-ans entre les Pays-Bas et la Monarchie catholique espagnole, et la guerre de Trente ans. Pendant ces années d'exil, les deux hommes se trouvèrent ainsi réunis autour d'un même projet politique : endiguer la puissance des Habsbourg en Europe et, autour d'une même controverse religieuse, départager deux mouvements calvinistes hollandais, les Remonstrants et les Contre-Remonstrants. La veine politique qui traverse tout l'album de Van de Venne est également évidente dans les dessins décrivant les jeux des gens simples et de la bourgeoisie, ainsi que dans ceux montrant le roi exilé et le Stadhouder. On les voit jouant au mail (fig. 14), dans une variante dont le but est de lancer la balle vers le poteau à l'arrière-plan, représenté juste à côté du clocher de la Kloosterkerk de La Haye qui, de ce fait, semble ici devenir la cible. Cette église était le quartier général des Contre-Remontrants dont l'artiste et le roi de Bohème 


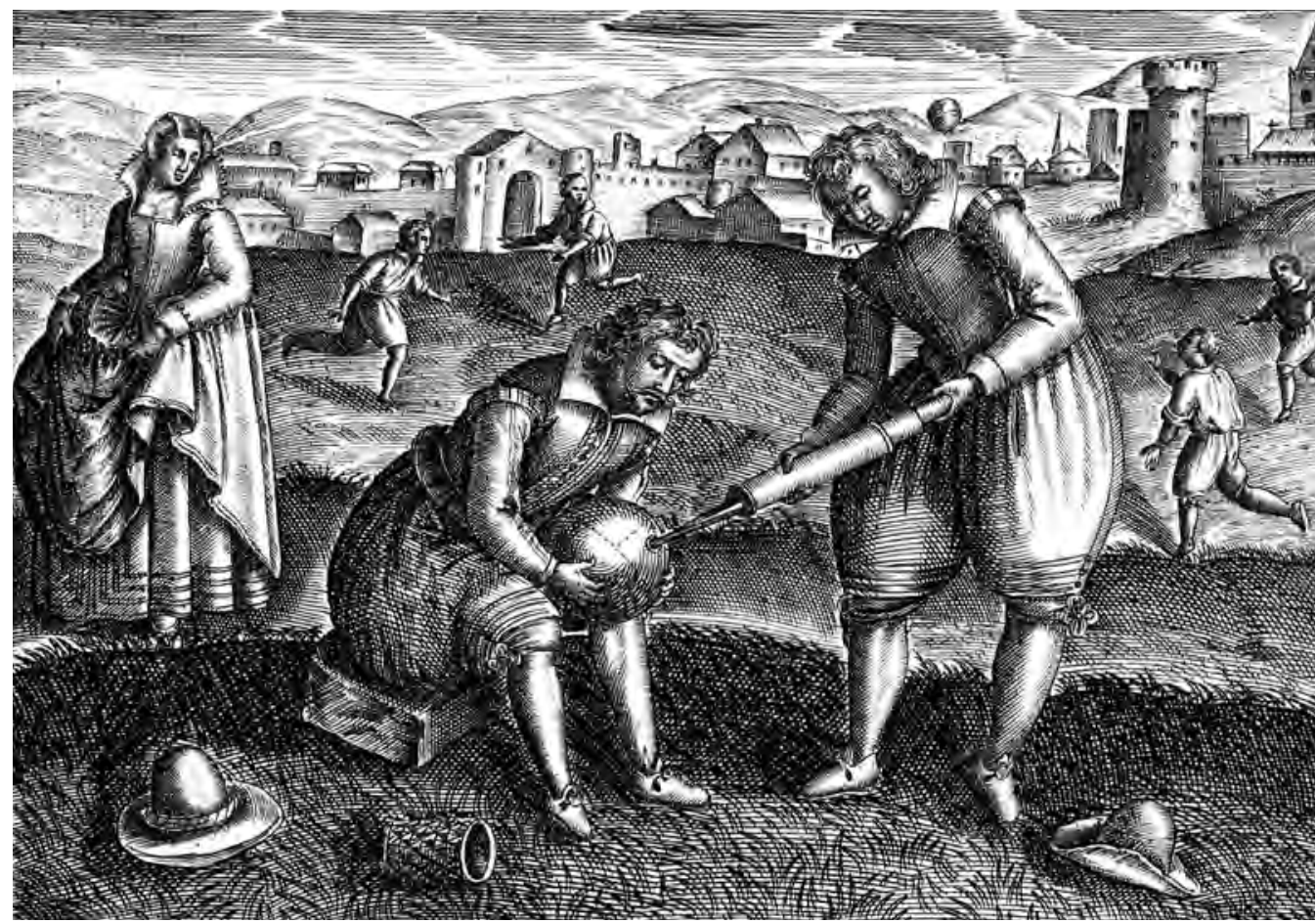

13. Crispijn de Passe (atelier), Jeu de Balle, 1600-1637,

Londres,

The British

Museum,

inv. $n^{\circ} 1873$,

0614.97 .

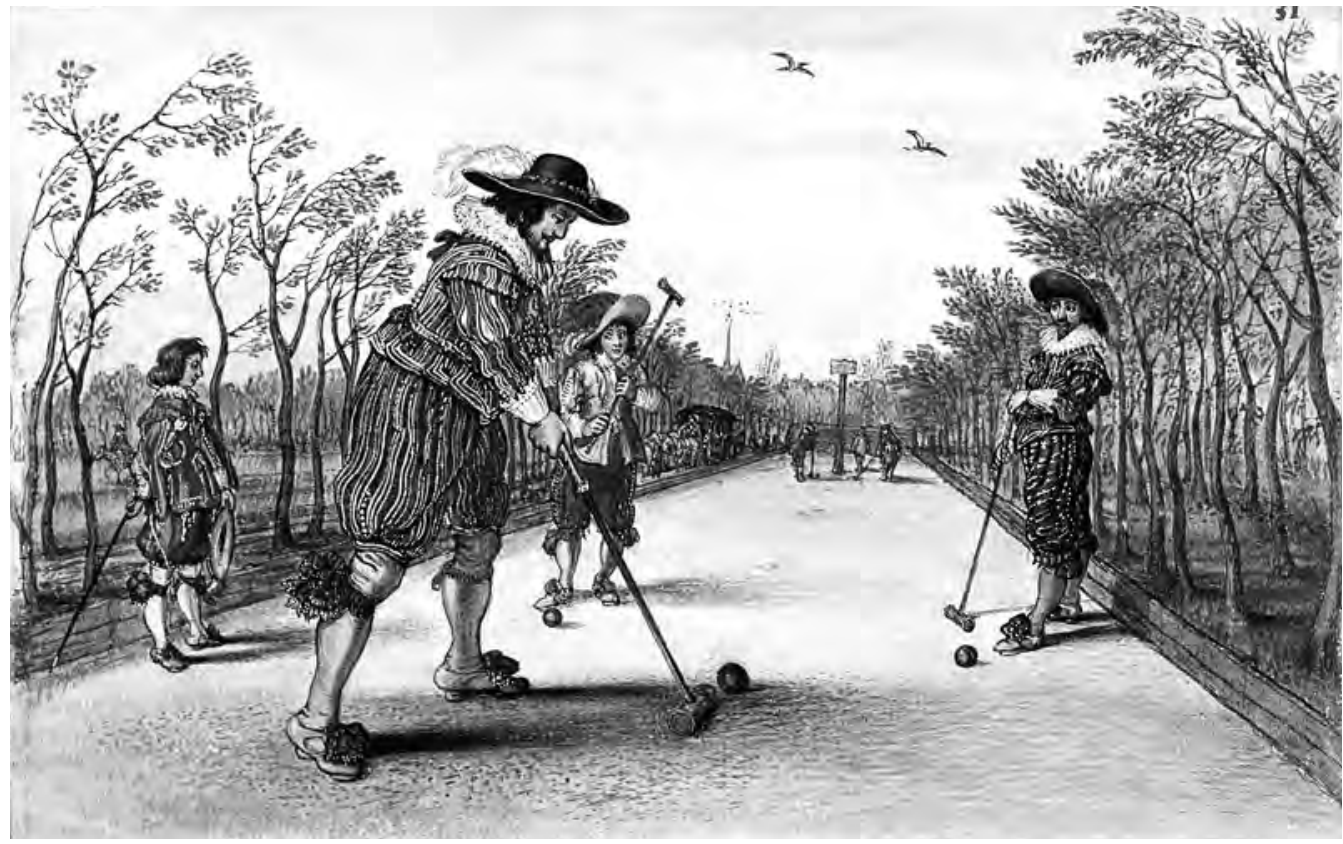

14. Adriaen Van de Venne, Le Mail, aquarelle sur pierre noire, rehauts d'argent, dans un album, v. $1620-1626$, Londres, The British Museum, inv. $\mathrm{n}^{\circ} 1978$, 0624.42.30 
étaient de fervents partisans, contrairement à l'indécis Frédéric-Henri qui, en 1625, avait tenté de se rapprocher des Remonstrants. L'église tient lieu ici de symbole des positions politiques et religieuses des deux joueurs : le roi s'apprête à frapper un coup sûr et bien orienté tandis que le Stadhouder tient une posture hésitante et timide. La portée politique d'une telle partie de balle paraît bien plus générale : dans la vie politique comme dans les loisirs sportifs, les deux hommes jouaient avec les mêmes objectifs.

Ces dispositifs visuels font ainsi du sport un agent où vis politica, vis moralis et vis erotica s'entremêlent. Et en cela, ces représentations fondent un imaginaire qui n'est pas si étranger à celui qui, depuis la fin du XIX ${ }^{e}$ siècle, caractérise la relation entre images et sports.

\section{Enfin... l'homme prémoderne faisait-il déjà du sport?}

Si cette culture visuelle prémoderne participe du renouvellement des normes et des pratiques ludico-sportives, elle offre aussi quelques éléments pour répondre à la question : pouvait-on réellement être un "sportif " avant le XVIII ${ }^{e}$ siècle ? Regarder avec plus d'attention certaines représentations visuelles peut permettre d'apporter quelques éléments de réponse. Parmi les dix petites gravures avec des scènes de la vie à Florence qui entourent le plan de la ville d'après Stefano Buonsignori (vers 1630), plusieurs présentent des épreuves sportives à la fois spécialisées et organisées. L'une montre un tournoi de lutte où deux couples d'athlètes se défient sur un ring surélevé, dressé dans la cour d'un palais (fig. 15) ; les spectateurs entourent l'estrade et, pour les plus nobles, observent par les fenêtres. Dans une seconde vignette, c'est une course de bateaux sur l'Arno qui rassemble, le long des berges et sur un pont, un public socialement plus diversifié. Avec les autres vues florentines, ces sujets ont une valeur représentative de la vie sociale et civique de la ville, et sont peut-être liés à des festivités princières, si l'on en croit les drapeaux médicéens qui flottent dans certaines scènes. Le caractère rituel ou officiel qui semble distinguer ces compétitions sportives est, en revanche, inexistant dans Les Lutteurs que Michael Sweerts peint en 1658 à Rome (fig. 16) : la lumière contrastée, le geste effrayé de l'homme levant les bras et fuyant, suggèrent la tension dramatique d'une rixe. Or, la scène présente deux athlètes lors d'un tournoi de lutte qui n'a rien d'improvisé : d'une part, l'organisation et la supervision de l'affrontement sont assurées par un jury dont les membres, coiffés d'un chapeau noir, sont visibles assis à une table à l'arrière-plan ; d'autre part, on note la présence d'un public populaire dont une partie est installée dans les tribunes.

15. Stefano Buonsignori (d'après), Lutteurs à Florence, 1627-1636, Londres, The British Museum, inv. $\mathrm{n}^{\circ} 1888$ 0612.67

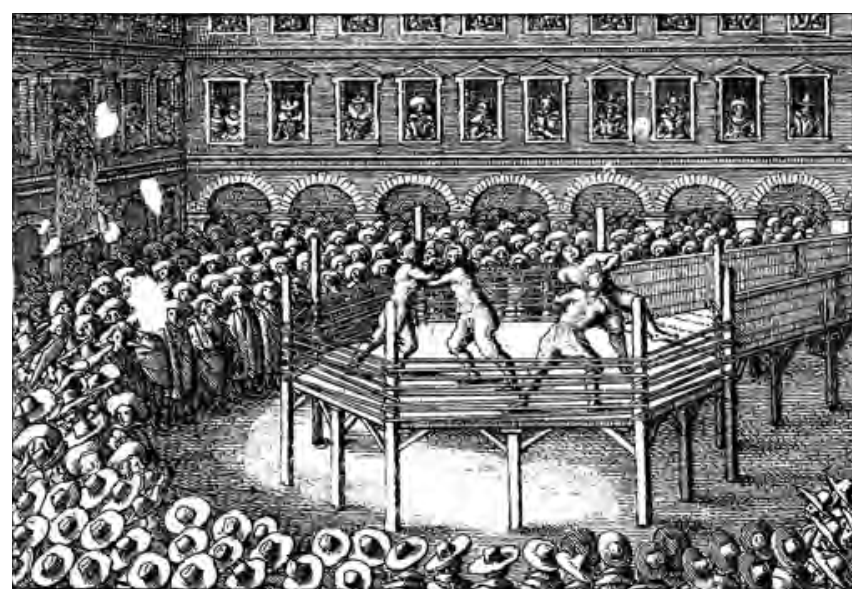

Pendant que les deux lutteurs se mesurent au corps à corps, à gauche, un troisième homme se déshabille pour entrer dans la compétition. Entre ce dernier et les deux compétiteurs, à l'arrière-plan, un quatrième athlète est agenouillé devant le jury (pour recevoir la récompense de sa victoire ?). La tonalité dramatique que l'artiste insuffle à la scène suggère la tension qui anime habituellement un tournoi, mais les compétitions sportives ayant lieu dans l'espace 
public faisaient peut-être aussi l'objet, à Rome comme ailleurs, de politiques visant à leur limitation ou à leur encadrement afin d'éviter désordres et violences (FENECH KROKE, 2016). Les sources textuelles sont riches à ce propos mais elles gardent la mémoire aussi, déjà au XVI ${ }^{\mathrm{e}}$ siècle, d'athlètes professionnels engagés précisément pour leurs compétences physiques et leurs performances dans le cadre de rencontres voyant s'affronter des quartiers ou bien

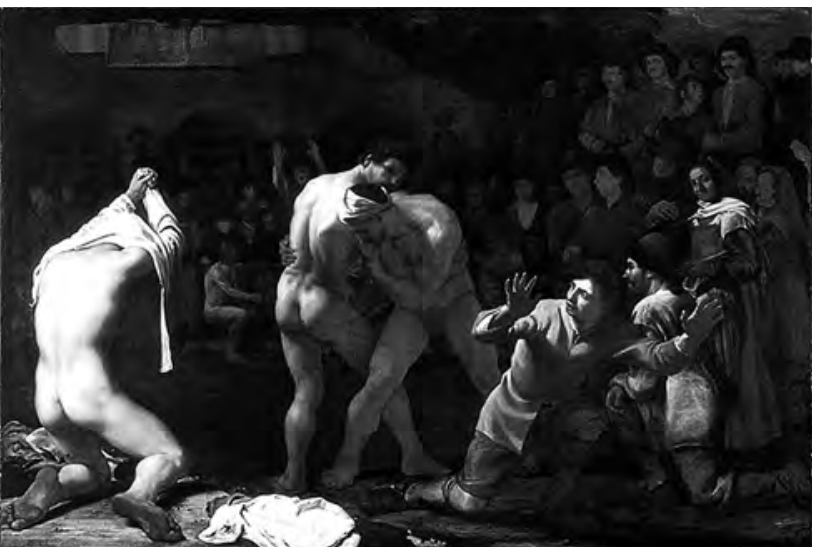

16. Michael Sweerts, Lutteurs romains, Karlsruhe Staatliche Kunsthalle, inv. 2496. des villes. Ainsi, si le sport se définit par la professionnalisation des acteurs, par les pratiques et les compétitions organisées, et par le développement d'une économie spécialisée, les jeux physiques prémodernes sont bien, in nuce, des sports. Cette hypothèse est moins une affirmation théorique catégorique qu'une provocation adressée à ceux pour lesquels les images seraient uniquement des objets esthétiques et non, comme nous le pensons, des sources au régime d'expression spécifique, qui jouent un rôle actif dans la construction d'imaginaires et de faits culturels et contribuent aussi à leur compréhension. L'essor contemporain de ces pratiques sportives et de leurs images a marqué, en premier lieu, la conception du temps et de son utilisation. Pour la culture de la première modernité, en effet, le temps libre est une donnée conceptuelle problématique : puisqu'on ne pense plus que le temps doit être consacré uniquement à Dieu, comme dans les siècles précédents (Le Goff), l'idée qu'il peut faire l'objet d'une utilisation individuelle, non religieuse et non productive se fait jour. C'est sous l'autorité d'Aristote que prend forme alors la notion moderne (et positive) de passetemps, participant à la mutation profonde que connaissent les concepts de divertissement et de plaisir, ce qui conduit à attribuer à certains jeux sportifs une utilité pour le corps et pour l'âme ${ }^{21}$. Cette valeur positive et instrumentale de l'exercice physique transforme sa perception : il n'est plus seulement vu comme le " lieu » du gaspillage du temps par l'individu et, en conséquence, de l'épanouissement des vices, mais comme le remède efficace contre ces derniers, et ce par l'introduction des notions de récréation et de conservation de la santé. En effet, la santé du corps physique devient alors le reflet de la santé du corps social. Si les images rendent compte de ce "temps affranchi ", de l'attention portée à la conservation de la santé du corps et de l'esprit, elles suggèrent aussi une répartition renouvelée de la sociabilité et enfin, en filigrane, des formes de mixité sociale : lorsque l'on joue, les contextes socioéconomiques deviennent plus poreux, attestant ainsi une circulation des pratiques sportives et une promotion sociale par celles-ci.

Donner formes aux pratiques, remodeler les goûts et les usages, influencer ainsi non seulement les modes de vie et les actes mais aussi la valeur que l'on donne à ces derniers : dès lors les images des sports prémodernes n'ont pas uniquement des fonctions informative, esthétique et, dans certains cas, injonctive. Elles ont également une efficace normative en tant qu'elles ont participé à façonner et à renouveler à la fois la manière de concevoir et de disposer du temps et du corps, les modes de vie collective et, enfin, les pratiques que les sociétés d'ancien régime ont commencé à penser comme souhaitables, voire désirables. 


\section{Notes}

1. CAILlOIS, 1967, p. 11.

2. Voir entre autres BENVÉNISTE, 1947 ; FINK, 1966 et 1968 ; WINNICOTT, 1975 ; ELIAS, DUNNING, 1986.

3. Je renvoie aux nombreuses contributions de G. Ortalli, à ses initiatives à la Fondazione Benetton (Treviso) et à la revue Ludica dont il est le fondateur.

4. BASCETTA, 1978 ; SANTACROCE, 1979 . ARIÈS, MARGOLIN, 1982 ; BURKE, 1995 ; BELMAS, 2006 ; MEHL, 2010 ; TURCOT, 2016.

5. KRÜGER, MCCLELLAND, 1984 ; ARCANGELI, 2010 et 2017 ; MCCLELLAND, 2003 2007 et 2009 ; MCCLELLAND, MERRILEES, 2009 ; MALLINCKRODT, SCHATTNER, 2016.

6. ULLMANN, 1977 ; GUTTMANN, 1978 ; VIGARELLO, 1988, 2002 et 2005.

7. Ce genre d'images n'a, jusqu'à aujourd'hui, attiré l'attention que de quelques rares historiens de l'art qui ont privilégié une approche strictement iconographique (BARLETTA, 1993 ; FRANCIONI, 1993 ; VAN SASSE VAN YSSELT, 1993 ; Arte e sport, 2004). Mentionnons cependant deux exceptions notables : l'essai sur le calcio florentin d'Horst Bredekamp qui, en partant d'une eau-forte de Jacques Callot (Calcio sur piazza Santa Croce, dans Capricci di varie figure, 1617) a révélé comment ce jeu, sa littérature et ses images ont constitué un enjeu politique majeur dans l'Italie de la Renaissance (BREDEKAMP, 1993). Cees de Bondt a également apporté une contribution stimulante à la connaissance du jeu de paume et de son rôle dans la culture de la Renaissance (DE BONDT, 1993, 2004 et 2006 ; voir aussi TOSI, 2013).

8. Voir, en particulier, BLÜME, 2004. Il s'agit d'un thème qui, réélaborant l'iconographie des "métiers des planètes " des manuscrits astrologiques arabes, se codifie et se développe à la fin du Moyen Âge et à la Renaissance : la représentation de la planète (anthropomorphe et sur un char de triomphe) y est accompagnée par les figures de ceux qui, étant nés sous son influence astrale, seraient prédisposés à l'exercice d'activités spécifiques.

9. Dans les deux cas c'est la raquette du volant. Dans le cas du Portrait de Charles IX la raquette pourrait toutefois être un rajout postérieur.

10. Marco Zoppo, Album, Londres, The British Museum, inv. 1920,0214.1.1.

11. Jan Amos Comenius, Opera Didactica Omnia, Amsterdam, 1657 ; idem, Orbis
Sensualium pictus / Die Sichtbare Welt, Nuremberg, 1658.

12. C'est le cas, par exemple, de l'Illustrissimi Wirtenbergici ducalis novi collegii (16081609) gravé par Ludwig Diztinger d'après les dessins de J. Ch. Neyffer ou encore de l'Academia sive speculum vitz scolasticx de Crispijn de Passe (1612, Johannes Janssonius).

13. Paris, BnF, Ea 79 Rés/63. La date pourrait être fictive et peut-être aussi le rôle de Guillaume Le Bé : je remercie Thierry Depaulis pour ces informations.

14. Heures de la famille Ango ou Livre des enfants, 1514, Paris, BnF, département des Manuscrits, NAL 392.

15. Antonio Scaino, Trattato del giuoco della palla, Venise, 1555, III, chap. VI ; traduction de l'auteur.

16. " [Le jeu convient] aux capitaines valeureux [qui] peuvent en tirer beaucoup de profit pour déployer leurs armées, pour ordonner une bataille, assiéger et défendre une forteresse, avancer dans le camp ennemi, et battre en retraite au bon moment $[\ldots]$, car la nature de ce jeu est si excellente que, même si ceux qui le pratiquent sont nombreux, rares sont ceux qui parviennent à être parfaits. C'est la raison pour laquelle il n'est adapté qu'à des hommes adroits, agiles, lestes et possédant une intelligence élevée et perspicace, qui savent prendre des décisions; et il faut que celles-ci, sans perdre de temps et promptement, viennent au secours des mains et de l'intellect d'un excellent joueur de balle " : Antonio Scaino, 1555, cité n. 15, p. 14, traduction de l'auteur.

17. Paris, BnF, département des Estampes et de la Photographie, Réserve OA-39-4 et Reserve OA-17-FOL.

18. Julia L. Hairston, Walter Stephens (dir.), The Body in Early Modern Italy, Baltimore, Johns Hopkins University Press, 2010 ; Linda Calof, William Bynum (dir.), A Cultural History of the Human Body. III. In the Renaissance, Oxford, Berg, (2010) 2014.

19. Henri Bergson, L'Évolution créatrice, Paris, Félix Alcan, 1907.

20. Énigme joyeuse pour les bons esprits, Paris, Jean Le Clerc, (vers 1615) 1624 (3 édition), p. 43.

21. Pensons par exemple aux passages que Robert Burton consacre à la question : Anatomie de la Mélancolie (1565), G. Venet (éd.), Paris, Gallimard, 2005 , p. 240-246.

\section{Bibliographie}

- ANGLo, 2011 : Sidney Anglo, L'escrime, la danse et l'art de la guerre. Le livre et la représentation du mouvement, Paris, Bibliothèque nationale de France, 2011.

- ARCANGELI, 1999 : Alessandro Arcangeli, " Play and Health in Medical Literature ", dans De zeventiende eeuw, n 15 , 1999, p. 3-11.

- ARCANGELI, 2003 : Alessandro Arcangeli, Recreation in the Renaissance. Attitude Towards Leisure and Pastimes in European Culture, c. 1425-1675, New York, Palgrave Macmillan, 2003 [éd. orig. : Passatempi rinascimentali. Storia culturale del divertimento in Europa (secoli XV-XVII), Rome, 2004].

- ARCANGELI, 2010 : Alessandro Arcangeli, "Sports ", dans Anthony Grafton, Glenn Most et Salvatore Settis (dir.), The Classical Tradition, Cambridge, MA, Harvard University Press, 2010, p. 905-906.

- ARCANGELI, 2014 : Alessandro Arcangeli, "Medicine, Gymnastic and Renaissance Sense of Body's Limits ", dans M. Mercedes Palandri and Angela Teja (dir.), Corpo e senso del limite/Sport and a Sense of the Body's Limits, Hanovre, NISH, 2014, , p. 357-362.

- ARCANGELI, 2017 : Alessandro Arcangeli, « Exercice and Leisure. Sport, Dance and Games ", dans William Caferro (dir.), The Routledge History of the Renaissance, Londres / New York, Routledge, 2017.

- ARIÈs, MARGOLIN, 1982 : Philippe Ariès et Jean-Claude Margolin (dir.), Les Jeux à la Renaissance, actes du colloque (Tours, XXIII ${ }^{\mathrm{e}}$ Colloque international d'études humanistes, 1980), Paris, Vrin, 1982.

- Arte e sport..., 2004 : Arte e sport a Firenze. Disegni e stampe dagli Uffizi, Florence, 2004.

- BARLETTA, 1993 : Chiara Barletta, « Iconografia del gioco nel Quattrocento ", dans Passare il tempo: la letteratura del gioco e dell'intrattenimento dal XII al XVI secolo, actes du colloque (Pienza, 1991), Rome, Salerno, 1993, vol. I, p. 239-250.

- BASCETTA, 1978 : Carlo Bascetta (dir.), Sport et giuochi. Trattati e scritti dal XV al XVIII secolo, Milan, Il Polifilo, 1978.

- BELMAS, 2006 : Élisabeth Belmas, Jouer autrefois. Essai sur le jeu dans la France moderne : XVI $-X V I I I^{e}$ siècle, Seyssel, Champ Vallon, 2006.

- BelmAs, 2011 : Élisabeth Belmas, " Jeux d'exercice, divertissement et virilité ", dans Georges Vigarello (dir.), Histoire de la virilité, 1. L'invention de la virilité. De l'Antiquité aux Lumières, Paris, Éditions du Seuil, 2011, p. 453-474.

- Benveniste, 1947 : Émile Benveniste, "Le jeu comme structure ", dans Deucalion, $\mathrm{n}^{\circ} 2$, 1947, p. 159-167. 
- BLÜME, 2004 : Dieter Blüme, " Children of the Planets: the Popularization of Astrology in 15th Century ", dans Micrologus, XI, 2004, p. 549-563.

- DE BONDT, 1993 : Cees de Bondt, Heefs yemant lust met bal of met recket te spelen? Tennis in Nederland tussen 1500 en 1800 , Amsterdam, Uitgeverij Verloren, 1993.

- DE Bondt, 2004 : Cees de Bondt, "Tiepolo's "The Death of Hyacinth" and the Image of the Game of Tennis in Art (1500-1800)", dans Studi veneziani, $\mathrm{n}^{\circ} 47,2004$, p. 281-403.

- DE BONDT, 2006 : Cees de Bondt, Royal Tennis in Renaissance Italy, Turnhout, Brepols, 2006

- BREDEKAMP, 1993 : Horst Bredekamp, Florentiner Fussball: die Renaissance der Spiele. Calcio als Fest der Medici, Francfort am Main, Campus, 1993.

- BRIOIST, DRÉVILLON, SERNA, 2002 : Pascal Brioist, Hervé Drévillon, Pierre Serna, Croiser le fer : violence et culture de l'épée dans la France moderne (XVI'-XVIII siècles), Seyssel, Champ Vallon, 2002.

- BURKe, 1995 : Peter Burke, «The Invention of Leisure in Early Modern Europe ", dans Past $\vartheta$ Present, $\mathrm{n}^{\circ}$ 146, 1995 , p. 136-150.

- CAILlois, 1958 : Roger Caillois, Les jeux et les hommes : le masque et le vertige, Paris, Gallimard, 1958.

- CAILLOIs, 1967 : Roger Caillois (dir.), Jeux et sports, Paris, Gallimard, 1967.

- CAPOROSSI, 2002 : Luisa Caporossi, " Gioco e tempo nell'appartamento dello specchio del Castello Estense a Ferrara ", dans Ludica, $\mathrm{n}^{\circ}$ 8, 2002, p. 98-114.

- CAstagnaro, 2009-2010 : Federica Castagnaro, "La scherma nei trattati italiani del Cinquecento: immagini e parole ", dans Ludica, nos 15-16, 2009-2010, p. 55-72.

- Crouzet-Pavan, 1993 : Élisabeth Crouzet-Pavan, "Quando la città si diverte. Giochi e ideologia urbana ", dans G. Ortalli (dir.), Gioco e giustizia nell'Italia di comune, Trévise, Viella, 1993, p. 35-48.

- Descola, 2017 : Philippe Descola, Cultures, Paris, INSEP / Carnets Nord / Le Pommier, 2017.

- ELIAS, DUNNING, (1986) 1994 : Norbert Elias et Eric Dunning, Sport et civilisation. La violence maîtrisée (1986), Josette Chicheporte et Fabienne Duvigneau (trad. fra.), Paris, Fayard, 1994.

- Fenech Kroke, 2014 : Antonella Fenech Kroke, "La palla da scanno en mots et en images : enquête sur un jeu à la Renaissance ", dans Revue d'histoire moderne et contemporaine, 2014/2, $\mathrm{n}^{\circ} 61-2$, p. 61-88.

- Fenech Kroke, 2014 : Antonella Fenech Kroke, "Michael Sweerts, Lutteurs romains", notice dans Francesca Cappelletti et Annick Lemoine (dir.), Les Bas-fonds du Baroque. La Rome du vice et de la misère, cat. exp. (Rome, Académie de France à Rome - Villa Médicis, 2014-2015/ Paris, Petit Palais-Musée des Beaux-arts de la Ville de Paris, 2015), Rome/Milan, Académie de France à Rome - Villa Médicis/Officina Libraria, 2014, p. 246-247.

- FENECH KROKE, à paraître : Antonella Fenech Kroke, " "Mattaccin tutti noi siamo". Giochi acrobatici e distorsioni corporee ", dans Francesco Lucioli et Francesca Aceto (dir.), Giocare tra medioevo ed età moderna. Modelli etici ed estetici per l'Europa, actes du colloque (Trévise, 2017, Fondazione Benetton Studi e Ricerche / CRH, EHESS / University College Dublin), Trévise, à paraître.

- FINK, 1966 : Eugen Fink, Le Jeu comme symbole du monde, Hans Hildenbrand et Alex Lindenberg (trad. fra.), Paris, Éditions de minuit, 1966.

- FINK, 1968 : Eugen Fink, "The Oasis of Happiness: Toward an Onthology of Play ", dans Yale French Studies, ${ }^{\circ} 41$, 1968, p. 19-30.

- Fontaine, 2009 : Marie-Madeleine Fontaine, "L'athlète et l'homme moderne : le regard nouveau de la Renaissance ", dans MCCLELLAND, MERRILEES, 2009, p. 115-145.

- FRANCIONI, 1993 : Stefania Francioni, "Iconografia del gioco nel Cinquecento ", dans Passare il tempo: la letteratura del gioco e dell'intrattenimento dal XII al XVI secolo, actes du colloque (Pienza, 1991), Rome, Salerno, 1993, vol. 1, p. 251-268. - FREDDI, 2017 : Laura Freddi, L'Educazione religiosa nella $\mathrm{Ca}^{\prime}$ Zoiosa di Vittorino da Feltre, Mantoue, 2017.

- GAGE, 2008 : Frances Gage, « Exercise for Mind and Body: Giulio Mancini, Collecting, and the Beholding of Landscape Painting in the Seventeenth Century ", dans Renaissance Quarterly, vol. 61,4, 2008, p. 1167-1207.

- GUERzoni, 1995 : Guido Guerzoni, "Playing Great Games: the Question of Giuoco in Italian 16th Century Courts ", dans Italian History and Culture, $\mathrm{n}^{\circ} 1$, 1995, p. 43-63.

- Guerzoni, 1996 : Guido Guerzoni, " "Ei non distingueva i giuochi patrizi da i plebei." Note sul gioco aristocratico e cortese tra Quattro e Cinquecento ", dans Ludica, $\mathrm{n}^{\circ} 2,1996$, p. 45-60.

- GutTMANN, 1978 : Allen Guttmann, From Ritual to Record: The Nature of Modern Sport, New York, Columbia University Press, 1978.

- Guttmann, 1986 : Allen Guttmann, Sport Spectators, New York, Columbia University Press, 1986.

- HuIzINGA, (1938) 1988 : Johan Huizinga, Homo ludens : essai sur la fonction sociale du jeu (1938), Cécile Sérésia (trad. fra.), Paris, Gallimard, 1988.

- KRÜGer, MCCLELLAND, 1984 : Arnd Krüger, John A. McClelland (dir.), Die Anfänge des modernen Sports in der Renaissance, Londres, Arena Publications, 1984.

- Lucchini, 1987 : Anna Lucchini, "Gli affreschi della sala dei giochi di Palazzo Borromeo ", dans Arte lombarda vol. 80/82, nos 1-3, 1987, p. 183-195.

- MALLINCKRodT, SchatTNeR, 2016 : Rebekka von Mallinckrodt, Angela Schattner (dir.), Sports and Physical Exercise in Early Modern Culture. New Perspectives on the History of Sports and Motion, actes du colloque (Londres, German Historical Institute, 2011), Abingdon, Oxon / New York, Routledge, 2016.

- MANSON, 2003 : Michel Manson, "L'image "malgré tout" dans les livres pour enfants du XVI ${ }^{\mathrm{e}}$ au milieu du XVIII ${ }^{\mathrm{e}}$ siècle ", dans Annie Renonciat (dir.), L'image pour enfants : pratiques, normes, discours (France et pays francophones, $X V I^{e}-X X^{e}$ siècles), Poitiers, La Licorne, 2003. - MCCLELland, 2002 : John A. McClelland, "Eros and Sport: A Humanist's Perspective ", dans Journal of Sport History, vol. 29, $\mathrm{n}^{\circ}$ 3, 2002, p. 395-406.

- McClelland, 2003 : John A. McClelland, "Ball Games, from Roman Gentleman to Renaissance Warrior ", dans James Anthony Margan, Militarism, Sport, Europe: War without Weapons, Londres / Portland, Frank Cass, 2003, p. 46-63.

- MCCLELLAND, 2007 : John A. McClelland, Body and Mind: Sport in Europe from the Roman Empire to the Renaissance, Londres, Routledge, 2007.

- MCCLELland, 2009 : John A. McClelland, "Sport" in Early Modern Europe? ", dans MCCLELLAND, MERRILEES, 2009, p. 23-40.

- MCClelland, MerRilees, 2009 : John A. McClelland, Brian S. Merrilees (dir.), Sport and Culture Sport and Culture in Early Modern Europe, Toronto, Center for Reformation and Renaissance Studies, 2009. 
- Menl, 2010 : Jean-Michel Mehl, Des jeux et des hommes dans la société médiévale, Paris, H. Champion, 2010.

- Merlotti, 2001 : Andrea Merlotti (dir.), Giochi di palla nel Piemonte medievale e moderno, Rocca de' Bardi, Centro studi storico-etnografici - Museo storico-etnografico della provincia di Cuneo "A. Doro ", 2001

- Morin, 2009 : Yvan Morin, "Conception du mouvement, de l'exercice, du jeu et du sport : de Marsile Ficin jusqu'au $\mathrm{XX}^{\mathrm{e}}$ siècle ", dans MCCLELlAND, MERRILEES, 2009, p. 316-389.

- ORTALLI, 1997 : Gherardo Ortalli, «The Origins of the Gambler-State: Licenses and Excises for Gaming Activities in the XIII and XIV Centuries (and the Case of Vicenza) ", dans Ludica, n 3, 1997, p. 108-131. - ORTALLI, 1999 : Gherardo Ortalli, « Lo stato e il giocatore: lunga storia di un rapporto difficile ", dans Giuseppe Imbucci (dir.), Il gioco pubblico in Italia: Storia, cultura e mercato, Venise, Marsilio, 1999, p. 33-43.

- RENSON, 1982 : Roland Renson, "Le jeu chez Juan Luis Vivès ", dans ARIÈS, MARGOLIN, 1982, p. 469-487.

- RIzzI, 2006 : Alessandra Rizzi, "Economia ed etica nella regolamentazione dell'azzardo nell'Italia tardomedievale ", dans Ludica, n 12, 2006, p. 64-80.

- ROMANI, 1995 : Andrea Marzio Romani, " "Honesto ocio post laborem ad reparandam virtutem quiete": corte, finanze e loisir nei ducati padani tra Cinque e Seicento ", dans S. Cavaciocchi (dir.), Tempo libero. Economia e società, secc. XIII-XVIII, Florence, Istituto Datini, 1995, p. 615-639. - RoyAlton-KISCH, 1988 : Martin Royalton-Kisch, Adriaen Van de Venne's Album, Londres, British Museum Publications, 1988.

- SANTACROCE, 1979 : Alberto Santacroce (dir.), Il gioco nella cultura moderna, Cosenza, Lerici, 1979.

- SCHMIDT, 2008 : Sandra Schmidt, Kopfübern und Luftspringen. Bewegung als Wissenschaft und Kunst in der Frühen Neuzeit, Munich, Fink, 2008.

- Schmidt, 2009 : Sandra Schmidt, " "Trois dialogues de l'exercice de sauter, et voltiger en l'air": Strategies of Ennoblement of a Bobily Practice in Sixteenth Century ", dans MCCLELlAnd, MERRILEES, 2009, p. 377-389.

- SCOTT BAKER, 2016 : Nicholas Scott Baker, " Deep Play in Renaissance Italy ", dans Mark Jurdjevic, Rolf Strøm-Olsen (dir.), Rituals of Politics and Culture in Early
Modern Europe: Essays in Honour of Edward Muir, Toronto, Centre for Reformation and Renaissance Studies, 2016, p. 259-281. - SIRAISI, 2003 : Nancy Siraisi, " History, Antiquarianism, and Medicine: The Case of Girolamo Mercuriale ", dans Journal of the History of Ideas, vol. 64, n ${ }^{\circ} 2,2003$, p. 231-251.

- TADDEI, 2001 : Ilaria Taddei, "L'encadrement des jeunes à Florence au $\mathrm{XV}^{\mathrm{e}}$ siècle ", dans Histoire urbaine, $\mathrm{n}^{\circ} 3$, 2001, p. 119-132.

- TORTAJAdA, 2008 : Maria Tortajada, "Évaluation, mesure, mouvement : la philosophie contre la science et les concepts de cinéma (Bergson, Marey) ", dans Revue européenne des sciences sociales, vol. XLVI, 2008, n ${ }^{\circ}$ 141, p. 95-111.

- Tosi, 2013 : Alessandro Tosi, « Tennis in Early Modern Visual Culture ", dans Nuncius. Journal of the Material and Visual History of Science, vol. 28, $\mathrm{n}^{\circ} 1,2013$, p. 85- 114 .

- TURCOT, 2016 : Laurent Turcot, Sport et loisirs. Une histoire des origines à nos jours, Paris, Gallimard, 2016.

- Ullmann, 1965 : Jacques Ullmann, De la gymnastique aux sports modernes, Paris, PUF, 1965.

- VAGENHeIM, 2008 : Ginette Vagenheim, "Una collaborazione tra antiquario e erudito: i disegni e le epigrafi di Pirro Ligorio al De Arte gymnastica di Mercurale ", dans Alessandro Arcangeli, Vivian Nutton (dir.), Girolamo Mercuriale, medicina e cultura nell'Europa del Cinquecento, actes du colloque (Forlì, 2006), Florence, Olschki, 2008, p. 127-157.

- VAGenheim, 2010 : Ginette Vagenheim, "Il contributo di Pirro Ligorio e di Piero Vettori al "De Arte Gymnastica" di Girolamo Mercuriale: il disegno del braccio col disco ", dans Mitteilungen des Kunsthistorischen Institutes in Florenz, vol. 54, $\mathrm{n}^{\circ} 1$, 2010-2012, p. 185-195.

- VAN SASSE VAN YSSELT, 1993 : Dorine Van Sasse Van Ysselt, "Il Calcio Fiorentino disegnato da Giovanni Stradano ", dans Mitteilungen des Kunsthistorischen Institutes in Florenz, vol. 37, n² 2/3, 1993, p. 481-487. - VAucelle, 2009a : Serge Vaucelle, " “L'Espée \& l'Esteuf". Escrime et Jeu de paume dans la France moderne $\left(\mathrm{XV}^{\mathrm{e}}\right.$ XVII ${ }^{\mathrm{e}}$ siècles) ", dans Sport History Review, $\mathrm{n}^{\circ} 40,2009$, p. 57-68.

- VAUCELLE, 2009b : " L'éducation corporelle des aristocrates français à l'âge classique : la place des traités didactiques ", dans MCClelland, Merrilees, 2009 , p. 247-266.
- Veldman, 2001 : Ilja M. Veldman, Profit and Pleasure: Print Books by Crispijn de Passe, Michael Hoyle (Engl. transl.), Rotterdam, Sound \& Vision Publishers, 2001.

- Veldman, 2004 : Ilja M. Veldman, " The Portrayal of Student Life and Universities in Early Modern Period ", dans Koen Goudriaan, Jaap van Moolenbroek, Ad Tervoort (dir.), Education and Learning in the Nederlands, 1400-1600. Essays in Honour of Hilde de Ridder-Symoens, Leyde, Brill, 2004, p. 315-338.

- VigARELlo, 1988 : Georges Vigarello, Une histoire culturelle du sport, techniques d'hier et d'aujourd'hui, Paris, Revue UPS / Robert Laffont, 1988.

- VigARELlo, 2002 : Georges Vigarello, Du jeu ancien au show sportif, la naissance d'un mythe, Paris, Éditions du Seuil, 2002. - VigARELlo, 2005 : Georges Vigarello, "S'exercer, jouer ", dans Idem (dir.), Histoire du corps, I. De la Renaissance aux Lumières, Paris, Éditions du Seuil, 2005. p. 245-317.

- WALKER, 1999 : Jonathan Walker, "Gambling and Venetian Noblemen, c. 1500-1700", dans Past e Present, $\mathrm{n}^{\circ} 162,1999$, p. 28-69.

- WeEmAns, 2002 : Michel Weemans, " Le modèle scopique. Regard et paysage chez Henri Bles ", dans Jacques Toussaint (dir.), Autour d'Henri Bles, actes du colloque (Maison de la Culture de la Province de Namur, 2000), Namur, Société archéologique, 2002, p. 211-225.

- WeEMANS, 2012 : Michel Weemans, "Quel rapport entre un jeu de paume et le roi David ? Analogie et exégèse visuelle dans le David et Bethsabée de Herri met de Bles ", dans Walter S. Melion, Lee Palmer Wandel (dir.), Early Modern Eyes, Leyde / Boston, Brill, 2012, p. 157-206. - WeEmans, 2013 : Michel Weemans, Herri met de Bles. Les ruses du paysage au temps de Bruegel et d'Érasme, Paris, Hasa, 2013.

- William, 2008 : James William, « Sport and the Elite in Early Modern England", dans Sport in History, vol. 28, n 3, 2008, p. 389-413.

- WinnicotT, 1975 : Donald Woods Winnicott, Jeu et réalité : l'espace potentiel, Claude Monod et J.-B. Pontalis (trad. fra.), Paris, Gallimard, 1975.

- ZEChER 2000 : Carla Zecher, "The Gendering of the Lute in Sixteenth-Century French Love Poetry ", dans Renaissance Quarterly, n³, 2000, p. 769-791. 